\title{
Information Geometry, Fluctuations, Non-Equilibrium Thermodynamics, and Geodesics in Complex Systems
}

\author{
Eun-jin Kim
}

check for

updates

Citation: Kim, E.-j. Information Geometry, Fluctuations,

Non-Equilibrium Thermodynamics, and Geodesics in Complex Systems. Entropy 2021, 23, 1393. https:// doi.org/10.3390/e23111393

Academic Editor: Miguel Rubi

Received: 29 September 2021

Accepted: 19 October 2021

Published: 24 October 2021

Publisher's Note: MDPI stays neutral with regard to jurisdictional claims in published maps and institutional affiliations.

Copyright: (c) 2021 by the authors. Licensee MDPI, Basel, Switzerland. This article is an open access article distributed under the terms and conditions of the Creative Commons Attribution (CC BY) license (https:// creativecommons.org/licenses/by/ $4.0 /)$.
Center for Fluid and Complex Systems, Coventry University, Priory St, Coventry CV1 5FB, UK; ejk92122@gmail.com

\begin{abstract}
Information theory provides an interdisciplinary method to understand important phenomena in many research fields ranging from astrophysical and laboratory fluids/plasmas to biological systems. In particular, information geometric theory enables us to envision the evolution of non-equilibrium processes in terms of a (dimensionless) distance by quantifying how information unfolds over time as a probability density function (PDF) evolves in time. Here, we discuss some recent developments in information geometric theory focusing on time-dependent dynamic aspects of non-equilibrium processes (e.g., time-varying mean value, time-varying variance, or temperature, etc.) and their thermodynamic and physical/biological implications. We compare different distances between two given PDFs and highlight the importance of a path-dependent distance for a time-dependent PDF. We then discuss the role of the information rate $\Gamma=\frac{d \mathcal{L}}{d t}$ and relative entropy in non-equilibrium thermodynamic relations (entropy production rate, heat flux, dissipated work, non-equilibrium free energy, etc.), and various inequalities among them. Here, $\mathcal{L}$ is the information length representing the total number of statistically distinguishable states a PDF evolves through over time. We explore the implications of a geodesic solution in information geometry for self-organization and control.
\end{abstract}

Keywords: information geometry; entropy; information rate; information length; fluctuations; Langevin equations; Fokker-planck equation; time-dependent probability density functions; self-organization

\section{Introduction}

Information geometry refers to the application of the techniques of differential geometry to probability and statistics. Specifically, it uses differential geometry to define the metric tensor that endows the statistical space (consisting of probabilities) with the notion of distance [1-31]. While seemingly too abstract, it permits us to measure quantitative differences among different probabilities. It then makes it possible to link a stochastic process, complexity, and geometry, which is particularly useful in classifying a growing number of data from different research areas (e.g., from astrophysical and laboratory systems to biosystems). Furthermore, it can be used to obtain desired outcomes $[6-10,15]$ or to understand statistical complexity [4].

For instance, the Wasserstein metric [6-10] was widely used in the optimal transport problem where the main interest is to minimize transport cost which is a quadratic function of the distance between two locations. It satisfies the Fokker-Planck equation for gradient flow which minimizes the entropy/energy functional [7]. For Gaussian distributions, the Wasserstein metric space consists of physical distances-Euclidean and positive symmetric matrices for the mean and variance, respectively (e.g., see [8]). 
In comparsion, the Fisher (Fisher-Rao) information [32] can be used to define a dimensionless distance in statistical manifolds [33,34]. For instance, the statistical distance $d s$ represents the number of indistinguishable states as $[5,33]$

$$
(d s)^{2} \equiv \sum_{j} \frac{d p_{j}^{2}}{p_{j}}=\sum_{j} p_{j}\left(d \ln p_{j}\right)^{2}=\sum_{j, \alpha, \beta} p_{j} \frac{\partial \ln p_{j}}{\partial \lambda^{\alpha}} \frac{\partial \ln p_{j}}{\partial \lambda^{\beta}} d \lambda^{\alpha} d \lambda^{\beta}=\sum_{\alpha, \beta} d \lambda^{\alpha} g_{\alpha \beta} d \lambda^{\beta} .
$$

Here the Fisher information metric $g_{\alpha \beta}=\left\langle\frac{\partial \ln p_{j}}{\partial \lambda^{\alpha}} \frac{\partial \ln p_{j}}{\partial \lambda^{\beta}}\right\rangle=\sum_{j} p_{j} \frac{\partial \ln p_{j}}{\partial \lambda^{\alpha}} \frac{\partial \ln p_{j}}{\partial \lambda^{\beta}}$ provides natural (Riemannian) distinguishability metric on the space of probability distributions. $\lambda^{\alpha \prime}$ s are the parameters of the probability $p_{j}$ and the angular brackets represent the ensemble average over $p_{j}$. Note that Equation (1) is given for a discrete probability $p_{j}$. For a continuous Probability Density Function (PDF) $p(x)$ for a variable $x$, Equation (1) becomes $(d s)^{2}=\int d x p(x)\left[d \ln (p(x)]^{2}=\sum_{\alpha, \beta} d \lambda^{\alpha} g_{\alpha \beta} d \lambda^{\beta}\right.$ where $g_{\alpha \beta}=\int d x p(x) \frac{\partial \ln (p(x))}{\partial \lambda^{\alpha}} \frac{\partial \ln (p(x))}{\partial \lambda^{\beta}}$.

For Gaussian processes, the Fisher metric is inversely proportional to the covariance matrices of fluctuations in the systems. Thus, in thermodynamic equilibrium, strong fluctuations lead to a strong correlation and a shorter distance between the neighboring states $[34,35]$. Alternatively, fluctuations determine the uncertainty in measurements, providing the resolution (the distance unit) that normalizes the distance between different thermodynamic states.

To appreciate the meaning of fluctuation-based metric, let us consider the (equilibrium) Maxwell-Boltzmann distribution $p\left(E_{j}\right)=\beta e^{-\beta E_{j}}$ for the energy state $E_{j}$

$$
\frac{p\left(E_{i}\right)}{p\left(E_{j}\right)}=e^{-\beta\left(E_{i}-E_{j}\right)}
$$

Here $\beta=1 / k_{B} T$ is the inverse temperature; $k_{B}$ is the Boltzmann constant; $T$ is the temperature of the heat bath. In Equation (2), the thermal energy $k_{B} T=\langle E\rangle$ of the heat bath (the width/uncertainty of the probability) provides the resolution to differentiate different states $\Delta E=E_{i}-E_{j}$. The smaller is the resolution (temperature), the more distinguishable states (more accessible information in the system) there are. It agrees with the expectation that a PDF gradient (the Fisher-information) increases with information [32].

This concept has been generalized to non-equilibrium systems [36-43], including the utilization for controlling systems to minimize entropy production [38,40,42], the measurement of the statistical distance in experiments to validate theoretical predictions [41], etc. However, some of these works rely on the equilibrium distribution Equation (2) that is valid only in or near equilibrium while many important phenomena in nature and laboratories are often far from equilibrium with strong fluctuations, variability, heterogeneity, or stochasticity [44-52]. Far from equilibrium, there is no (infinite-capacity) heat bath that can maintain the system at a certain temperature, or constant fluctuation level. One of the important questions far from equilibrium is indeed to understand how fluctuation level $\beta(t)^{-1}$ changes with time. Furthermore, PDFs no longer follow the Maxwell-Boltzmann nor Gaussian distributions and can involve the contribution from (rare) events of large amplitude fluctuations [53-62]. Therefore, the full knowledge of time-varying PDFs and the application of information geometry to such PDFs have become of considerable interest.

Furthermore, while in equilibrium [63,64], information theoretical measures (e.g., Shannon information entropy) can be given thermodynamic meanings (e.g., heat), in nonequilibrium such interpretations are not always possible and equilibrium thermodynamic rules can break down locally (e.g., see $[65,66]$ and references therein). Much progress on these issues has been made by different authors (e.g., [65-82]) through the development of information theory, stochastic thermodynamics, and non-equilibrium fluctuation theorems with the help of the Fisher information [32], relative entropy [83], mutual information $[84,85]$, etc. Exemplary works include the Landauer's principle which links information loss to the ability to extract work [86,87]; the resolution of Maxwell's demon paradox [88]; black hole thermodynamics [89,90]; various thermodynamic inequal- 
ity/uncertainty relations $[65,68,91-97]$; and linking different research areas (e.g., nonequilibrium processes to quantum mechanics [98-100], physics to biology [101]).

The paper aims to discuss some recent developments in the information geometric theory of non-equilibrium processes. Since this would undoubtedly span a broad range of topics, this paper will have to be selective and will focus on elucidating the dynamic aspect of non-equilibrium processes and thermodynamic and physical/biological implications. Throughout the paper, we highlight that time-varying measures (esp. variance) introduces extra complication in various relations, in particular, between the information geometric measure and entropy production rate. We make the efforts to make this paper self-contained (e.g., by including the derivations of some well-known results) wherever possible.

The remainder of this paper is organized as follows. Section 2 discusses different distances between two PDFs and the generalization for a time-dependent non-equilibrium PDF. Section 3 compares the distancs from Section 2. Section 4 discusses key thermodynamic relations that are useful for non-equilibrium processes. Section 5 establishes relations between information geometric quantities (in Section 2) and thermodynamics (in Section 4). In Section 6, we discuss the concept of a geodesic in information geometry and its implications for self-organization or designing optimal protocols for control. Conclusions are provided in Section 7.

\section{Distances/Metrics}

This section discusses the distance defined between two probabilities (Section 2.1) and along the evolution path of a time-dependent probability (Section 2.2). Examples and comparisons of these distances are provided in Section 3.1. For illustration, we use a PDF $p(x, t)$ of a stochastic variable $x$ and differential entropy $S=-\int d x p(x, t) \ln (p(x, t))$ by using the unit $k_{B}=1$.

\subsection{Distance Between Two PDFs}

We consider the distance between two PDFs $p_{1}=p\left(x, t_{1}\right)$ and $p_{2}=p\left(x, t_{2}\right)$ of a stochastic variable $x$ at two times $t_{1}$ and $t_{2}$, respectively where $t_{1}=t_{2}$ or $t_{1} \neq t_{2}$ in general.

\subsubsection{Wootters' Distance}

The Wootters' distance $[5,33]$ is defined in quantum mechanics by the shortest distance between the two $p_{1}$ and $p_{2}$ that have the wave functions $\psi_{1}$ and $\psi_{2}\left(p_{1}=\left|\psi_{1}\right|^{2}\right.$ and $\left.p_{2}=\left|\psi_{2}\right|^{2}\right)$, respectively. Specifically, for given $p_{1}$ and $p_{2}$, the distance $s\left(p_{1}, p_{2}\right)$ between $p_{1}$ and $p_{2}$ can be parameterized by infinitely many different paths between $p_{1}$ and $p_{2}$. Letting $z$ be the affine parameter of a path, we have

$$
s\left(p_{1}, p_{2}\right)=\int_{1}^{2} d s=\int d z \frac{d s(z)}{d z}=\int d z \sqrt{\sum_{\alpha, \beta} \frac{d \lambda^{\alpha}}{d z} g_{\alpha \beta} \frac{d \lambda^{\beta}}{d z}},
$$

where $d s$ is given in Equation (1). Among all possible paths, the minimum of $s\left(p_{1}, p_{2}\right)$ is obtained for a particular path that optimizes the quantum distinguishability; the (Hilbertspace) angle between the two wave functions provides such minimum distance as

$$
W\left[p_{1}, p_{2}\right]=\cos ^{-1}\left[\int d x\left[p\left(x, t_{1}\right)\right]^{\frac{1}{2}}\left[p\left(x, t_{2}\right)\right]^{\frac{1}{2}}\right] .
$$

Equation (4) is for a pure state and has been generalized to mixed states (e.g., see [37,102] and references therein). Note that the Wootters' distance is related to the Hellinger distance [43]. 


\subsubsection{Kullback-Leibler (K-L) Divergence/Relative Entropy}

Kullback-Leibler (K-L) divergence between the two PDFs [83], also called relative entropy, is defined by

$$
\mathcal{K}\left(p_{1} \mid p_{2}\right)=\int d x p\left(x, t_{1}\right) \ln \left(\frac{p\left(x, t_{1}\right)}{p\left(x, t_{2}\right)}\right) .
$$

Relative entropy quantifies the difference between a PDF $p_{1}$ and another PDF $p_{2}$. It takes the minimum zero value for identical two PDFs $p_{1}=p_{2}$ and becomes large as $p_{1}$ and $p_{2}$ become more different. However, as it is defined in Equation (5), it is not symmetric between $p_{1}$ and $p_{2}$ and does not satisfy the triangle inequality. It is thus not a metric in a strict sense.

\subsubsection{Jensen Divergence}

The Jensen divergence (also called Jensen distance) is the symmetrized KullbackLeibler divergence defined by

$$
\left.\mathcal{J}\left(p_{1} \mid p_{2}\right)=\frac{1}{2}\left[\mathcal{K}\left(p_{1} \mid p_{2}\right)+\mathcal{K}\left(p_{2} \mid p_{1}\right)\right)\right] .
$$

While the square root of the Jensen-Shannon divergence $\sqrt{\mathcal{J}\left(p_{1} \mid p_{2}\right)}$ is a metric $[4,103]$, $\mathcal{J}\left(p_{1} \mid p_{2}\right)$ itself has also been used in examining statistical complexity (e.g., see $\left.[43,104,105]\right)$.

\subsubsection{Euclidean Norm}

In analysis of big data, the Euclidean norm $[5,106]$ is used, which is defined by

$$
\left|p_{1}-p_{2}\right|^{2}=\int d x\left[p\left(x, t_{1}\right)-p\left(x, t_{2}\right)\right]^{2} .
$$

While Equation (7) has a direct analogy to the physical distance, it has a limitation in measuring statistical complexity due to the neglect of the stochastic nature [5]. For instance, the Wootters' distance in Equation (4) was shown to work better than Equation (7) in capturing complexity in the logistic map [5].

\subsection{Distance along the Path}

Equations (4)-(7) can be used to define the distance between the two given PDFs $p\left(x, t_{1}\right)$ and $p\left(x, t_{2}\right)$ at times $t_{1}$ and $t_{2}\left(t_{2}>t_{1}\right)$. However, $p(x, t)$ at the intermediate time $t=\left(t_{1}, t_{2}\right)$ can take an infinite number of different values depending on the exact path that a system takes between $p\left(x, t_{1}\right)$ and $p\left(x, t_{2}\right)$. One example would be i) $p\left(x, t_{1}\right)=$ $p\left(x, t_{2}\right)=p(x, t)$ for all $t=\left(t_{1}, t_{2}\right)$ and $x$, in comparison with ii) $p\left(x, t_{1}\right)=p\left(x, t_{2}\right)$ but $p(x, t) \neq p\left(x, t_{1}\right)$ and $p(x, t) \neq p\left(x, t_{2}\right)$. What is necessary is a path-dependent distance that depends on the exact evolution and the form of $p(x, t)$ for $t=\left(t_{1}, t_{2}\right)$.

\subsubsection{Information Rate}

Calculating a path-dependent distance for a time-dependent PDF $p(x, t)$ requires the generalization of the distance in Section 2.1. To this end, we consider two (temporally) adjacent PDFs along the trajectory, say, $p(x, t)$ and $p(x, t+d t)$ and calculate the (infinitesimal) relative entropy between them in the limit $d t \rightarrow 0$ to the leading order in $O(d t)$ :

$$
\begin{aligned}
& \lim _{d t \rightarrow 0} \frac{1}{(d t)^{2}} \mathcal{K}[p(x, t+d t) \mid p(x, t)]=\lim _{d t \rightarrow 0} \frac{1}{(d t)^{2}} \mathcal{K}[p(x, t) \mid p(x, t+d t)] \\
& =\lim _{d t \rightarrow 0} \frac{1}{(d t)^{2}} \mathcal{J}[p(x, t+d t) \mid p(x, t)]=\frac{1}{2} \int d x p(x, t)\left(\partial_{t} \ln p(x, t)\right)^{2} \equiv \frac{1}{2} \Gamma^{2} .
\end{aligned}
$$


Here, we used $p(x, t+d t)=p(x, t)+(d t) \partial_{t} p+\frac{1}{2}(d t)^{2}\left(\partial_{t} p\right)^{2}+\mathrm{O}\left((d t)^{3}\right), \ln (1+r)=$ $r-\frac{1}{2} r^{2}+\mathrm{O}\left(r^{3}\right)$ for $r \ll 1$, and $\int d x \partial_{t} p(x, t)=\int d x \partial_{t t} p(x, t)=0$ because of the total probability conservation $\int d x p(x, t)=1$. Due to the symmetry of $\mathcal{K}[p(x, t+d t) \mid p(x, t)]$ to leading order $\mathrm{O}\left((d t)^{2}\right), \mathcal{K}[p(x, t+d t) \mid p(x, t)]=\mathcal{J}[p(x, t+d t) \mid p(x, t)]$ to $\mathrm{O}\left((d t)^{2}\right)$.

In Equation (8), the information rate $\Gamma$ is defined by [15-29]

$$
\Gamma^{2}(t)=\lim _{d t \rightarrow 0} \frac{2}{(d t)^{2}} J[p(x, t+d t) \mid p(x, t)]=\int d x p(x, t)\left(\partial_{t} \ln p(x, t)\right)^{2}=4 \int d x\left(\partial_{t} q(t)\right)^{2} .
$$

Here, $q=\sqrt{p}$, and $\Gamma \geq 0$ by definition. We note that the last term in terms of $q$ in Equation (9) can be used when $q=p=0$. The dimensions of $\Gamma^{2} \equiv \mathcal{E}$ and $\Gamma$ are (time $)^{-2}$ and (time $)^{-1}$, respectively. They do not change their values under nonlinear, timeindependent transformation of variables (see Appendix A). Thus, using the unit where the length is dimensionless, $\mathcal{E}$ and $\Gamma$ can be viewed as the kinetic energy per mass and velocity, respectively. For this reason, $\Gamma$ was called the velocity (e.g., in $[15,17])$.

Note that $\mathcal{E}$ can be viewed as the Fisher information [32] if time is interpreted as a parameter (e.g., [97]). However, time in classical mechanics is a passive quantity that cannot be changed by an external control. $\Gamma$ is also called the entropy production rate in quantum mechanics [107]. However, as shown in Sections 4.1 and 4.4, the relation between $\Gamma$ and thermodynamic entropy production rate is more complicated (see Equation (28)).

$\Gamma$ in Equation (9) is the information rate representing how quickly new information is revealed as a PDF evolves in time. Here, $\Gamma^{-1}=\tau$ is the characteristic time scale of this information change in time. To show that $\Gamma$ is related to fluctuation's smallest time scale [97], we assume that $\lambda^{\alpha \prime}$ s are the estimators (parameters) of a $p(x, t)$ and use the CramérRao bound on the Fisher information $g_{\alpha \beta}=\int d x p(x, t) \partial_{\lambda^{\alpha}}[\ln p(x, t)] \partial_{\lambda^{\beta}}[\ln p(x, t)] \geq C_{\alpha \beta}^{-1}$ where $C^{\alpha \beta} \equiv\left\langle\delta \lambda^{\alpha} \delta \lambda^{\beta}\right\rangle$ is the covariance matrix (e.g., see [32]); $\delta \lambda^{\alpha}=\lambda^{\alpha}-\left\langle\lambda^{\alpha}\right\rangle$ denotes fluctuation. Using $\frac{d \ln p}{d t}=\frac{\partial \ln p}{\partial \lambda^{\alpha}} \frac{d \lambda^{\alpha}}{d t}$ then leads to

$$
\Gamma^{2}=\sum_{\alpha \beta} \frac{d \lambda^{\alpha}}{d t} g_{\alpha \beta} \frac{d \lambda^{\beta}}{d t} \geq \sum_{\alpha \beta} \frac{d \lambda^{\alpha}}{d t} C_{\alpha \beta}^{-1} \frac{d \lambda^{\beta}}{d t} .
$$

For the diagonal $g_{\alpha \beta}=g_{\alpha} \delta_{\alpha \beta}$, Equation (10) is simplified as

$$
\sum_{\alpha} \frac{1}{\left\langle\left(\delta \lambda^{\alpha}\right)^{2}\right\rangle}\left(\frac{d \lambda^{\alpha}}{d t}\right)^{2} \leq \Gamma^{2}
$$

Equation (11) shows how the RMS fluctuation-normalized rate at which the parameter $\lambda^{\alpha}$ can change is bounded above by $\Gamma$. If there is only $\alpha=1\left(\lambda^{\alpha}=\lambda \delta_{\alpha, 1}\right)$, Equation (11) is further simplified:

$$
\frac{1}{\sqrt{\left\langle(\delta \lambda)^{2}\right\rangle}}\left|\frac{d \lambda}{d t}\right| \leq \Gamma,
$$

clearly showing that $\lambda$ normalized by its RMS fluctuations cannot change faster than the information rate.

Finally, it is worth highlighting that Equation (9) is general and can be used even when the parameters $\lambda^{\alpha \prime}$ s and $g_{\alpha \beta}$ in $\Gamma^{2}$ in Equation (10) are unknown. Examples include the cases where PDFs are empirically inferred from experimental/observational data. Readers are referred to Refs. $[21,23,28]$ for examples. It is only the special case where we have a complete set of parameters $\lambda^{\alpha \prime}$ s of a PDF that we can express $\Gamma$ using the Fisher information as in Equation (10). For instance, for a Gaussian $p(x, t)$ that is fully described by the mean value $\langle x\rangle$ and variance $\frac{1}{2 \beta},\left(\lambda^{1}, \lambda^{2}\right)=(\langle x\rangle, \beta)$. 


\subsubsection{Information Length}

Since $\Gamma \propto \sqrt{\mathcal{J}[p(x, t+d t) \mid p(x, t)]}$ is a metric [103] as noted in Section 2.1, $\Gamma$ is also a metric. Thus, we sum $\Gamma$ along the trajectory to define a finite distance. Specifically, starting with an initial PDF $p(x, t=0)$, we integrate $\Gamma(t)$ over time to obtain the dimensionless number as a function time as

$$
\mathcal{L}(t)=\int_{0}^{t} d t_{1} \Gamma\left(t_{1}\right)
$$

$\mathcal{L}$ is the information length [15-31] that quantifies the total change in information along the trajectory of $p(x, t)$ or the total number of statistically distinguishable states it evolves through over time. [We note that different names (e.g., statistical length [108], or statistical distance [97]) were also used for $\mathcal{L}$.] It is important to note that unlike the Wootters' distance (the shortest distance among all possible paths between the two PDFs) in Equation (3) (e.g., [5]), $\mathcal{L}(t)$ in Equation (13) is fixed for a given time-evolving PDF $p(x, t)$.

By definition in Equation (13), $\mathcal{L}(t=0)=0$ and $\mathcal{L}(t)$ monotonically increases with time since $\Gamma \geq 0$ (e.g., see Figure A2 in [22]). $\mathcal{L}(t)$ takes a constant value only in a stationary state $\left(\Gamma=\partial_{t} p=0\right)$. One of its important consequences is that when $p(x, t)$ relaxes into a stationary PDF in the long time limit $t \rightarrow \infty, \Gamma(t) \rightarrow 0$ and $\mathcal{L}(t) \rightarrow \mathcal{L}_{\infty}$ as $t \rightarrow \infty$ where $\mathcal{L}_{\infty}$ is a constant depending on initial conditions and parameters. This property of $\mathcal{L}_{\infty}$ was used to understand attractor structure in a relaxation problem; specifically, Refs. $[15,16,18,22,28]$ calculated $\mathcal{L}_{\infty}$ for different values of the mean position $x_{0}$ of an initial PDF and examined how $\mathcal{L}_{\infty}$ depends on $x_{0}$. Furthermore, $\Gamma$ and $\mathcal{L}$ were shown to be useful for quantifying hysteresis in forward-backward processes [19], correlation and self-regulation among different players [23,25], and predicting the occurrence of sudden events [27] and phase transitions $[23,25]$. Some of these points are illustrated in Section 3.1.

\section{Model and Comparison of Metrics}

For discussing/comparing different metrics in Section 2 and statistical measures in Section 4, we use the following Langevin model [109]

$$
\frac{d x}{d t}=f(x, t)+\xi=-\partial_{x} V(x, t)+\xi
$$

Here, $V(x, t)$ is, in general, a time-dependent potential which can include an internal potential and an external force; $\xi$ is a short (delta)-correlated Gaussian noise with the following statistical property

$$
\left\langle\xi(t) \xi\left(t^{\prime}\right)\right\rangle=2 D \delta\left(t-t^{\prime}\right) .
$$

Here, the angular brackets represent the ensemble average over the stochastic noise $\xi$; $D \geq 0$ is the amplitude of $\xi$. It is important to note that far from equilibrium, the average (e.g., $\langle x(t)\rangle$ ) is a function of time, in general.

The exact PDFs can be obtained for the Ornstein-Uhlenbeck (O-U) process which has $V=\frac{\gamma}{2}(x-v(t))^{2}$ and $f=-\gamma(x-v(t))$ in Equation (14). Here, $v(t)$ is a deterministic function of time. Specifically, for the initial Gaussian PDF $p(x, 0)$

$$
p(x, 0)=\sqrt{\frac{\beta_{0}}{\pi}} e^{-\beta_{0}\left(x-x_{0}\right)^{2}},
$$

a time-dependent PDF remains Gaussian at all time:

$$
\begin{aligned}
p(x, t) & =\int d x_{1} p\left(x, t ; x_{1}, 0\right) p\left(x_{1}, 0\right)=\sqrt{\frac{\beta}{\pi}} e^{-\beta(x-\langle x\rangle)^{2}}, \\
\frac{1}{2 \beta(t)} & =\frac{e^{-2 \gamma t}}{2 \beta_{0}}+\frac{D\left(1-e^{-2 \gamma t}\right)}{\gamma},
\end{aligned}
$$




$$
\langle x(t)\rangle=x_{0} e^{-\gamma t}+\gamma \int_{0}^{t} d t_{1} e^{-\gamma\left(t-t_{1}\right)} v\left(t_{1}\right) .
$$

In Equations (16)-(19), $x_{0}=\langle x(t=0)\rangle, \beta_{0}=\beta(t=0)$, and $\left\langle(\delta x)^{2}\right\rangle=\frac{1}{2 \beta}=\sigma^{2}$. Here, $\beta, \sigma$ and $\sigma^{2}$ are the inverse temperature, standard deviation, and variance, respectively; $\beta_{0}$ and $x_{0}$ are the values of $\beta$ and $\langle x\rangle$, respectively, at $t=0$. Equation (18) shows that as $t \rightarrow \infty, \beta(t \rightarrow \infty)=\frac{\gamma}{2 D}$. Note that we use both $\beta$ and $\sigma$ here to clarify the connections to the previous works $[15,17,22,26-28]$.

\subsection{Geometric Structure of Equilibrium/Attractors}

To elucidate the main difference between the distances in Equations (4)-(6) and (13), we consider the relaxation problem by assuming $v(t)=0$. In the following, we compare the distance between $p(x, 0)$ and $p(x, t \rightarrow \infty)$ by using $p_{1}\left(x, t_{1}\right)=p(x, 0)$ and $p_{2}\left(x, t_{2}\right)=$ $p(x, t \rightarrow \infty)$ in Equations (4)-(6) and Equation (13). Analytical expressions for these distances are given in [22].

Each curve in Figure 1 shows how each distance depends on the initial mean position $x_{0}$. The four different curves are for $\mathcal{L}_{\infty}$ (in blue), Wootters' distance (in orange), K-L relative entropy (in green), and Jensen divergence (in red), respectively. The relative entropy and Jensen divergence exhibit similar behavior, the red and green color curves being superimposed on each other. Of note is a linear relation between $\mathcal{L}_{\infty}$ and $x_{0}$ in Figure 1. Such linear relation is not seen in other distances. This means that the information length is a unique measure that manifests a linear geometry around its equilibrium point in a linear Gaussian process [28,30]. Note that for a nonlinear force $f, \mathcal{L}_{\infty}$ has a power-law relation with $x_{0}$ for a sufficiently large $x_{0}[18,28]$. These contrast with the behaviour in a chaotic system $[16,28]$ where $\mathcal{L}_{\infty}$ depends sensitively on the initial condition and abruptly changes with $x_{0}$. Thus, the information length provides a useful tool to geometrically understand attractor structures in relaxation problems.

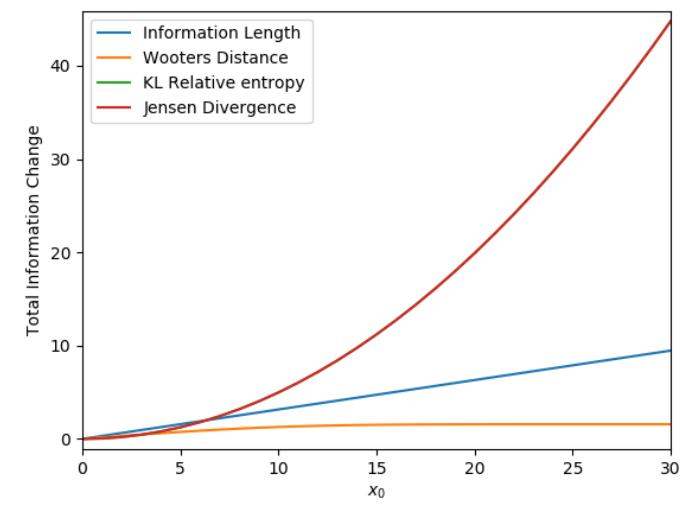

Figure 1. The distance against $x_{0}$ between $p(x, 0)$ and $p(x, t \rightarrow \infty)$ for the O-U process. (Figure 1 in [22]).

\subsection{Correlation between Two Interacting Components}

We next show that the information length is also useful in elucidating the correlation between two interacting species such as two competing components relaxing to the same equilibrium in the long time limit. Specifically, the two interacting components with the time-dependent PDFs $P_{1}(x, t)$ and $P_{2}(x, t)$ are coupled through the Dichotomous noise $[110,111]$ (see Appendix $B$ ) and relax into the same equilibrium Gaussian PDF $P_{1}(x, t \rightarrow \infty)=P_{2}(x, t \rightarrow \infty)=\frac{1}{2} P(x, t \rightarrow \infty)$ around $x=0$ in the long time limit. Here, $P(x, t)=P_{1}(x, t)+P_{2}(x, t)$ is the total PDF. For the case considered below, $P(x, t)$ satisfies the O-U process (see Appendix B for details). We choose the initial conditions where $P_{1}(t=0)=P_{1}(t \rightarrow \infty)$ with zero initial mean value while $P_{2}(t=0)$ takes an initial mean value $x_{0}$. These are demonstrated in the cartoon figure, Figure 2a,c. 

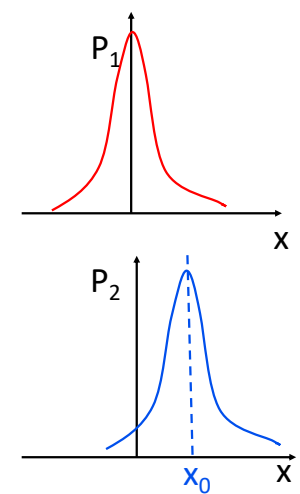

(a) $t=0$
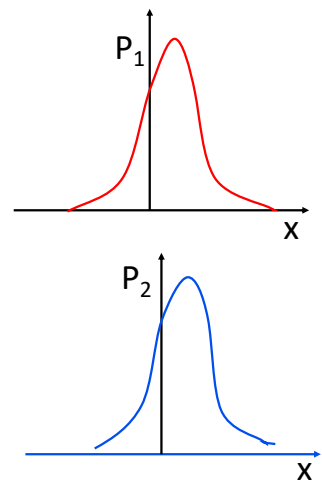

(b) $0<t<\infty$
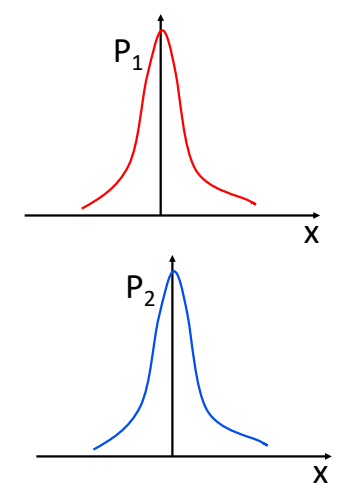

(c) $t=\infty$

Figure 2. $P_{1}$ (top) and $P_{2}$ (bottom) at time $t=0$ in panel (a), $t=(0, \infty)$ in panel (b), and $t \rightarrow \infty$ in panel (c). Note that $P_{1}(0<t<\infty) \neq P_{1}(t=0)\left(=P_{1}(t \rightarrow \infty)\right)$.

Although $P_{1}(t=0)=P_{1}(t \rightarrow \infty)$, at the intermediate time $t=(0, \infty), P_{1}(x, t)$ evolves in time due to its coupling to $P_{2}$ and thus $P_{1}(x, t) \neq P_{1}(x, t=0)$, as shown in Figure $2 \mathrm{~b}$. Consequently, $\mathcal{L}(t)$ calculated from $P_{1}$ monotonically increases to its asymptotic value $\mathcal{L}_{\infty}$ until it reaches the equilibrium (see Figure A2 in [22] for time-evolution of $\mathcal{L}$ from $P_{1}$ and $P_{2}$ ). On the other hand, $P_{2}$ with an initial mean value $x_{0}$ undergoes a different time evolution (unless $x_{0}=0$ ) until it reaches the equilibrium.

The distances in Equations (4)-(7) and (13) can be calculated from the total $P=$ $P_{1}+P_{2}, P_{1}$ and $P_{2}$ for different values of $x_{0}$. Results are shown in Figure 3a-c, respectively; (a) $P(x, 0)$ and $P(x, t \rightarrow \infty),\left(\right.$ b) $P_{1}(x, 0)$ and $P_{1}(x, t \rightarrow \infty)$, and (c) $P_{2}(x, 0)$ and $P_{2}(x, t \rightarrow \infty)$, respectively. Specifically, for each value of $x_{0}$, we calculate the distances in Equations (4)-(7) and (13) by using $p_{1}\left(x, t_{1}\right)=P(x, 0)$ and $p_{2}\left(x, t_{2}\right)=P(x, t \rightarrow \infty)$ for Figure 3a; $p_{1}\left(x, t_{1}\right)=P_{1}(x, 0)$ and $p_{2}\left(x, t_{2}\right)=P_{1}(x, t \rightarrow \infty)$ for Figure $3 \mathrm{~b} ; p_{1}\left(x, t_{1}\right)=$ $P_{2}(x, 0)$ and $p_{2}\left(x, t_{2}\right)=P_{2}(x, t \rightarrow \infty)$ for Figure $3 c$. The same procedure above is then repeated for many other $x_{0}$ 's to show how each distance depends on $x_{0}$.

(a)

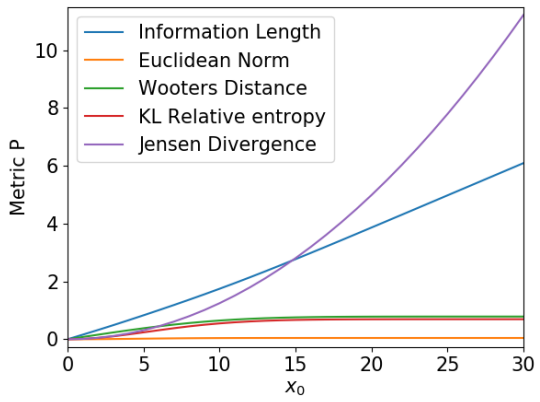

(b)

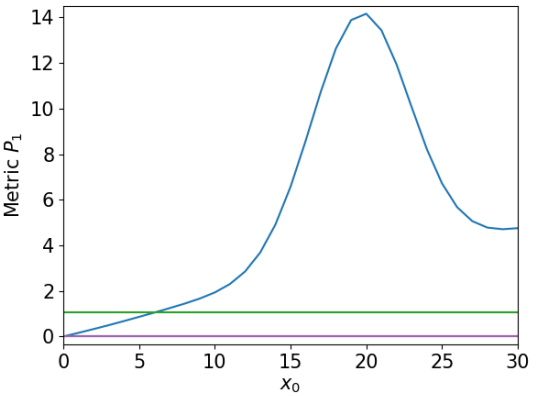

(c)

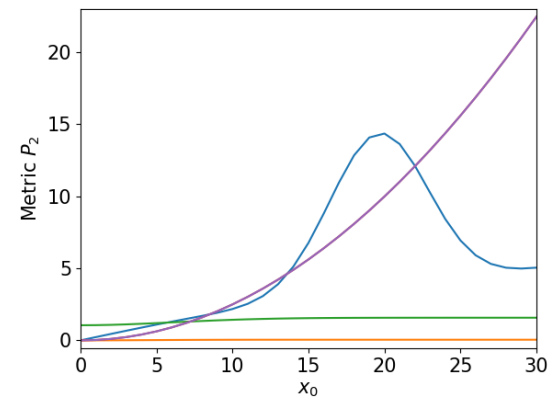

Figure 3. The distance between $P(x, 0)$ and $P(x, t \rightarrow \infty)$ against in $x_{0}$ in $(\mathbf{a}) ; P_{1}(x, 0)$ and $P_{2}(x, t \rightarrow \infty)$ in $(\mathbf{b}) ; P_{2}(x, 0)$ and $P_{2}(x, t \rightarrow \infty)$ in (c). (Figure 4 in [22]).

For the total $P$, a linear relation between $\mathcal{L}_{\infty}$ and $x_{0}$ is seen in Figure 3a (like in Figure 1). This linear relation is not seen in $\mathcal{L}_{\infty}$ calculated from either $P_{1}$ or $P_{2}$ in Figure $3 \mathrm{~b}$ or Figure 3c; a non-monotonic dependence of $\mathcal{L}_{\infty}$ in Figure $3 b, c$ is due to large-fluctuations and strong-correlation between $P_{1}$ and $P_{2}$ during time-evolution for large $x_{0}$. What is quite remarkable is that in contrast to other distances, $\mathcal{L}_{\infty}$ calculated from $P_{1}$ and $P_{2}$ in Figure $3 b, c$ exhibits a very similar dependence on $x_{0}$. It means that despite very different time-evolutions of $P_{1}$ and $P_{2}$ (see Figure 2), they undergo similar total change in information. These results suggest that strong coupling between two components can be inferred from their similar information length (see also [24,25]). 


\section{Thermodynamic Relations}

To elucidate the utility of information geometric theory in understanding non-equilibrium thermodynamics, we review some of the important thermodynamic measures of irreversibility and dissipation [112] and relate them to information geometric measures $\Gamma$ and $\mathcal{K}$ [29]. For illustration below, we use the model in Equations (14) and (15) unless stated otherwise. Corresponding to Equations (14) and (15) is the following Fokker-Planck equation [109]

$$
\frac{\partial p(x, t)}{\partial t}=-\frac{\partial}{\partial x}[f(x, t) p(x, t)]+D \frac{\partial^{2} p(x, t)}{\partial x^{2}}=-\partial_{x} J(x, t),
$$

where $J=f p-D \partial_{x} p$ is the probability current.

\subsection{Entropy Production Rate and Flow}

For non-equilibrium thermodynamics, we need to consider the entropy in the system $S$ and the environment $S_{m}$, and the total entropy $S_{T}=S+S_{m}$. To clarify the difference among these, we go over some derivation by using $\partial_{t} p=-\partial_{x} J$ and $J=f p-D \partial_{x} p$ to obtain

$$
\dot{S}=\frac{d S(x, t)}{d t}=-\int d x \partial_{t} p \ln p=\frac{d S_{T}(x, t)}{d t}-\frac{d S_{m}(x, t)}{d t},
$$

where,

$$
\dot{S}_{T}=\frac{d S_{T}}{d t}=\int d x\left(\frac{1}{D p} J^{2}\right), \quad \dot{S}_{m}=\frac{d S_{m}}{d t}=\int d x\left(\frac{1}{D} J f\right) .
$$

Here, we used integration by parts in $t$ and $x . \dot{S}_{T}$ denotes the (total) entropy production rate, which is non-negative $\dot{S}_{T} \geq 0$ by definition, and serves as a measure of irreversibility [112]. The sign of $\dot{S}_{m}$ in Equation (22) represents the direction in which the entropy flows between the system and environment. Specifically, $\dot{S}_{m}>0\left(\dot{S}_{m}<0\right)$ when the entropy flows from the system (environment) to the environment (system). $\dot{S}_{m}$ is related to the heat flux $Q=D S_{m}$ from the system to the environment. The equality $\dot{S}_{T}=0$ holds in an equilibrium reversible process. In this case, $\dot{S}=-\dot{S}_{m}=-\frac{Q}{D}$, which is the usual equilibrium thermodynamic relation. In comparison, when $\dot{S}=0, \dot{S}_{T}=\dot{S}_{m} \geq 0$.

For the O-U process with $V=\frac{\gamma}{2}(x-v(t))^{2}$ and $f=-\gamma(x-v(t))$ in Equations (14), (17)-(19), (21) and (22) lead to (see [29] for details)

$$
\begin{aligned}
S(t) & =\frac{1}{2}\left[1+\ln \frac{\pi}{\beta}\right], \\
D \dot{S_{T}} & ==\frac{\left(\partial_{t} \beta\right)^{2}}{8 \beta^{3}}+\left(\partial_{t}\langle x\rangle\right)^{2}=\left(\partial_{t} \sigma\right)^{2}+\left(\partial_{t}\langle x\rangle\right)^{2}, \\
\dot{S} & =-\frac{\partial_{t} \beta}{2 \beta}=\frac{\partial_{t} \sigma}{\sigma} \\
\dot{S}_{m} & =\dot{S}_{T}-\dot{S} .
\end{aligned}
$$

Here, we used $J=[f+2 D \beta(\delta x)] p=\left[-\frac{\partial_{t} \beta}{2 \beta}(\delta x)+\partial_{t}\langle x\rangle\right] p, \partial_{x} p=-2 \beta(\delta x) p, f=$ $-\gamma(x-v(t)), \partial_{t}\langle x\rangle=\langle f\rangle, 2 D \beta-\gamma=-\frac{\partial_{t} \beta}{2 \beta}$, and $\frac{\partial_{t} \beta}{\beta}=-2 \frac{\partial_{t} \sigma}{\sigma}$.

In order to relate these thermodynamical quantities $\dot{S}_{T}$ and $\dot{S}$ above to the information rate $\Gamma$, we recall that for the $\mathrm{O}-\mathrm{U}$ process $[15,17,26-28]$,

$$
\mathcal{E}=\Gamma^{2}=2 \beta\left(\partial_{t}\langle x\rangle\right)^{2}+\frac{\left(\partial_{t} \beta\right)^{2}}{2 \beta^{2}}=\frac{1}{\sigma^{2}}\left[2\left(\partial_{t} \sigma\right)^{2}+\left(\partial_{t}\langle x\rangle\right)^{2}\right] .
$$


Equations (24) and (27) then give us

$$
\Gamma^{2}=\frac{D}{\sigma^{2}} \dot{S}_{T}+\dot{S}^{2} .
$$

Interestingly, Equation (28) reveals that the entropy production rate needs be normalized by variance $\sigma^{2}$. This is because of the extensive nature of $\dot{S}_{T}$ unlike $\Gamma$ or $\dot{S}$. That is, $\dot{S}_{T}$ changes its value when the variable $x$ is rescaled by a scalar factor, say, $\alpha(>0)$ as $y=\alpha x$. Furthermore, Equation (28) shows that the information rate $\Gamma$ in general does not have a simple relation to the entropy production rate (c.f., [107]).

One interesting limit of Equation (28) is the case of constant $\beta(t)$ with $\dot{S}=0$. In that case, Equation (24) becomes $D \dot{S}_{T}=\left(\partial_{t}\langle x\rangle\right)^{2}$ while Equations (13), (27) and (28) give us

$$
\begin{aligned}
\mathcal{L}(t) & =\frac{\langle x(t)\rangle-\langle x(t=0)\rangle}{\sigma}, \\
\Gamma^{2} & =\frac{1}{\sigma^{2}}\left(\partial_{t}\langle x\rangle\right)^{2}=\frac{D}{\sigma^{2}} \dot{S}_{T} .
\end{aligned}
$$

Equation (29) simply states that $\mathcal{L}$ measures the total change in the mean value normalized by fluctuation level $\sigma$. Equation (30) manifests a linear relation between $\Gamma^{2}$ and $\dot{S}_{T}$ when $\partial_{t} \sigma=0$, as invoked in the previous works (e.g., [107]). Furthermore, a linear relation between $\Gamma^{2}$ and $\dot{S}_{T}$ in Equation (30) implies that minimizing the entropy production $\int^{t} d t_{1} \dot{S}_{T}$ along the trajectory corresponds to minimizing $\int_{0}^{t} d t_{1} \Gamma^{2}\left(t_{1}\right)$, which, in turn, is equivalent to minimizing $\mathcal{L}(t)$ (see Section 5 for further discussions).

Finally, to demonstrate how entropy production rate and thermal bath temperature $(D)$ are linked to the speed of fluctuations $c=\sigma \Gamma$ [97], we rewrite Equation (28) as

$$
c=\sigma \Gamma=\left[D \dot{S}_{T}+\sigma^{2} \dot{S}^{2}\right]^{\frac{1}{2}} .
$$

For constant variance $\dot{\beta}=0$, Equation (31) gives a simple relation $c=\sigma \Gamma=\sqrt{D \dot{S}_{T}}$.

\subsection{Non-Equilibrium Thermodynamical Laws}

To relate the statistical measures in Section 4.1 to thermodynamics, we let $U$ (internal energy) be the average potential energy $U=\langle V\rangle$ and obtain (see also $[66,113]$ and references therein)

$$
\frac{d U}{d t}=\frac{d}{d t}\langle V\rangle \equiv \dot{W}-\dot{Q}
$$

where

$$
\begin{aligned}
\dot{W} & =\int d x\left(\partial_{t} V\right) p=\left\langle\partial_{t} V\right\rangle, \\
\dot{Q} & =-\int d x V\left(\partial_{t} p\right)=\int d x J f=\langle f \dot{x}\rangle=D \dot{S}_{m} .
\end{aligned}
$$

The power $\dot{W}$ represents the average rate at which the work is done to the system because of time-varying potential; the average work during the time interval $\left[t_{0}, t\right]$ is calculated by $W=\int_{t_{0}}^{t} d t^{\prime} \dot{W}\left(t^{\prime}\right)$. On the other hand, $\dot{Q}$ represents the rate of dissipated heat.

Equation (32) establishes the non-equilibrium thermodynamic relation $\dot{U}=\dot{W}-\dot{Q}$. Physically, it simply means that the work done to the system $\dot{W}$ increases $U$ while the dissipated heat to the environment $\dot{Q}$ decreases it. Equations (21), (32), and (34) permit us to define a non-equilibrium (information) free energy $\mathcal{F}(t)=U(t)-D S(t)$ [92] and its time-derivative

$$
\dot{\mathcal{F}}=\dot{U}-D \dot{S}=\dot{W}-D \dot{S}_{T},
$$


where $\dot{\mathcal{F}}=\frac{d \mathcal{F}}{d t}$ and $\dot{U}=\frac{d U}{d t}$. Since $\dot{S}_{T} \geq 0$, Equation (35) leads to the following inequality

$$
D \dot{S}_{T}=\dot{W}-\dot{\mathcal{F}} \equiv \dot{W}_{D} \geq 0,
$$

where the non-negative dissipated power (lost to the environment) $\dot{W}_{D}$ is defined. Finally, the time-integral version of Equation (36) provides the bound on the average work performed on the system as $W-\Delta \mathcal{F}=W_{D} \geq 0$ (e.g., [68]).

\subsection{Relative Entropy as a Measure of Irreversibility}

The relative entropy has proven to be useful in understanding irreversibilities and non-equilibrium thermodynamic inequality relations [91-94,114-116]. In particular, the dissipated work $W_{D}=W-\Delta \mathcal{F}$ (in Equation (36)) is related to the relative entropy between the PDFs in the forward and reverse processes

$$
W_{D}=D \mathcal{K}\left[p_{F}\left(\gamma_{F}(t)\right) \mid p_{R}\left(\gamma_{R}(t)\right)\right] .
$$

(e.g., see [91-94].) Here, $p_{F}\left(\gamma_{F}(t)\right)$ and $p_{R}\left(\gamma_{R}(t)\right)$ are the PDFs for the forward and reverse processes driven by the forward $\gamma_{F}(t)$ and reverse $\gamma_{R}(t)$ protocols, respectively. Using Equation (36) in Equation (37) immediately gives

$$
\dot{S}_{T}=\frac{d}{d t} \mathcal{K}\left[p_{F}\left(\gamma_{F}(t)\right) \mid p_{R}\left(\gamma_{R}(t)\right)\right] \geq 0,
$$

which is a proxy for irreversibility (see $[115,116]$ for a slightly different expression of Equation (38)). It is useful to note that forward and reversal protocols are also used to establish various fluctuations theorems for different dissipative measures such as entropy production, dissipated work, etc. (see, e.g., [80] for a nice review and references therein).

However, we cannot consider forward and reversal protocols in the absence of a model control parameter that can be prescribed as a function of time. Even in this case, the relative entropy is useful in quantifying irreversibility through inequalities, and this is what we focus on in the remainder of Section 4.3.

To this end, let us consider a non-equilibrium state $p(x, t)$ which has an instantaneous non-equilibrium stationary state $p_{s}(x, t)$ and calculate the relative entropy between the two. Here, $p_{s}(x, t)$ is a steady solution of the Fokker-Planck equation $\partial_{t} p_{s}=0$ in Equation (20) (e.g., see [29]). Specifically, one finds $p_{s}(x, t)=e^{-\frac{V(x, t)-\mathcal{F}_{s}(t)}{D}}$ by treating the parameters to be constant (being frozen to their instantaneous values at a given time). Here, $V$ and $\mathcal{F}_{s}$ are the potential energy and the stationary free energy, respectively. For clarity, an example of $p_{s}(x, t)$ is given in Section 4.4.

The average of $\ln p_{s}(t)$ in the non-equilibrium state $p(x, t)$ can be expressed as follows:

$$
\int d x p(x, t) \ln p_{s}(x, t)=-\frac{1}{D} \int d x p(x, t)\left(V(x, t)-\mathcal{F}_{S}(t)\right)=-\frac{1}{D}\left(U(t)-\mathcal{F}_{S}(t)\right) .
$$

Equations (35) and (39) then give us

$$
\mathcal{F}(t)-\mathcal{F}_{s}(t)=D \int d x p(x, t) \ln \left[\frac{p(x, t)}{p_{s}(x, t)}\right] \equiv D \mathcal{K}\left[p(x, t) \mid p_{s}(x, t)\right] \geq 0 .
$$

Here, we used the fact the relative entropy is non-negative. Equation (40) explicitly shows that non-equilibrium free energy is bounded below by the stationary one $\mathcal{F} \geq \mathcal{F}_{s}$ (see also [1,92] and references therein for open Hamiltonian systems).

Equation (40) together with Equation (35) then lead to the following irreversible work $W_{\text {irr }}[29,92]$ :

$$
W_{\text {irr }} \equiv W-\Delta \mathcal{F}_{s}=D \Delta S_{T}+\Delta\left(\mathcal{F}-\mathcal{F}_{s}\right)=D \Delta S_{T}+D \Delta \mathcal{K}\left[p(x, t) \mid p_{s}(x, t)\right] .
$$


Here, $\Delta \mathcal{K}\left[p(x, t) \mid p_{s}(x, t)\right]=\mathcal{K}\left[p(x, t) \mid p_{s}(x, t)\right]-\mathcal{K}\left[p\left(x, t_{0}\right) \mid p_{s}\left(x, t_{0}\right)\right]$, etc. The derivation of Equation (41) for open-driven Hamiltonian systems is provided in [92] (see their Equation (38)).

On the other hand, we directly calculate the time-derivative of $\mathcal{K}\left[p(x, t) \mid p_{s}(x, t)\right]$ in Equation (40) by using $p_{s}(x, t)=e^{-\frac{V(x, t)-\mathcal{F}_{S}(t)}{D}}, \dot{S}_{T}=\dot{S}+\dot{S}_{m}, \int d x p \partial_{t} V=\dot{W}$ and $\dot{Q}=$ $-\int d x \partial_{t} p V=D \dot{S}_{m}$, and $W_{i r r}=W-\Delta \mathcal{F}_{s}$ :

$$
\frac{d}{d t} \mathcal{K}\left[p(x, t) \mid p_{s}(x, t)\right]=-\dot{S}+\frac{1}{D} \frac{d}{d t}\left[\int d x V p-\mathcal{F}_{S}\right]=-\dot{S}_{T}+\frac{1}{D}\left[\dot{W}-\frac{d}{d t} \mathcal{F}_{S}\right] .
$$

One can see easily that equating Equation (42) to $\frac{1}{D}\left[\dot{\mathcal{F}}-\dot{\mathcal{F}}_{s}\right]$ (from Equation (40)) simply recovers $\dot{W}-\frac{d}{d t} \mathcal{F}=D \dot{S}_{T}$ in Equation (35).

Finally, we obtain a differential form of Equation (41) by using $\dot{W}_{i r r}=\dot{W}-\frac{d}{d t} \mathcal{F}_{s}$ in Equation (42) as follows

$$
\dot{W}_{i r r}=D \dot{S}_{T}+D \frac{d}{d t} \mathcal{K}\left[p(x, t) \mid p_{s}(x, t)\right]
$$

\subsection{Example}

We consider $v(t)=u t$ with a constant $u$ so that $V=-\frac{\gamma}{2}(x-u t)^{2}$ in Equation (14). While the discussion below explicitly involves $v(t)$, the results are general and valid for the limiting case $v(t)=0$. The case with $v(t)=0$ is an example where the forward and reversal protocols do not exist while a non-equilibrium stationary state does.

For $f=-\gamma(x-u t)$, Equation (19) is simplified as follows

$$
\langle x(t)\rangle=x_{0} e^{-\gamma t}+u t-\frac{u}{\gamma}\left(1-e^{-\gamma t}\right) .
$$

For the non-equilibrium stationary state with fixed $\gamma$ and $D, \beta_{s}=\frac{\gamma}{2 D}$ is also constant $\left(\frac{d}{d t} \mathcal{F}_{s}=0\right)$. Therefore, we have

$$
p_{s}(x, t)=\sqrt{\frac{\beta_{s}}{\pi}} e^{-\beta_{s}(x-u t)^{2}} .
$$

Then, we can find (see [29] for details)

$$
\begin{aligned}
\mathcal{K}\left[p(x, t) \mid p_{s}(x, t)\right] & =\frac{1}{2}\left[-1+\ln \left(\beta / \beta_{s}\right)\right]+\beta_{s}\left((\langle x\rangle-u t)^{2}+\frac{1}{2 \beta}\right), \\
D \dot{S}_{T} & =\left[\left(-\gamma\left\langle x_{0}\right\rangle e^{-\gamma t}+u\left(1-e^{-\gamma t}\right)\right)^{2}+\frac{1}{2 \beta}(2 \beta D-\gamma)^{2}\right], \\
\dot{Q} & =\left[-\gamma\left\langle x_{0}\right\rangle e^{-\gamma t}+u\left(1-e^{-\gamma t}\right)\right]^{2}-\frac{\gamma}{2 \beta}(2 \beta D-\gamma), \\
\dot{W} & =-u \int d x \gamma(x-u t) p=-u \gamma\langle x-u t\rangle=u^{2}\left(1-e^{-\gamma t}\right), \\
\frac{d}{d t} \mathcal{K}\left[p(x, t) \mid p_{s}(x, t)\right] & =-\frac{1}{D}\left[\left(\partial_{t}\langle x\rangle\right)^{2}+\left(\partial_{t} \sigma\right)^{2}-u \partial_{t}\langle x\rangle\right]=-\dot{S}_{T}+\frac{1}{D} \dot{W} .
\end{aligned}
$$

Here, we used Equations (23)-(26), $\langle x\rangle$ and $\beta$ in Equations (44) and (18), respectively, and $\dot{W}=\langle-\gamma u(x-u t)\rangle=u \partial_{t}\langle x\rangle$.

It is worth looking at the two interesting limits of Equations (46)-(50). First, in the long time limit as $t \rightarrow \infty$, the following simpler relations are found:

$$
\begin{aligned}
& \langle x\rangle \rightarrow u\left(t-\gamma^{-1}\right), 2 \beta \rightarrow \frac{\gamma}{D}=2 \beta_{s}, \\
& D \dot{S}_{T}=\dot{Q}=\dot{W}=(\sigma \Gamma)^{2} \rightarrow u^{2},
\end{aligned}
$$




$$
\dot{S} \rightarrow 0, \frac{d}{d t} \mathcal{K}\left[p(x, t) \mid p_{s}(x, t)\right] \rightarrow 0 .
$$

Equation (51) illustrates how the external force $v(t)=u t \neq 0$ keeps the system out of equilibrium even in the long time limit, with non-zero entropy production and dissipation. When there is no external force $u=0$, the system reaches equilibrium as $t \rightarrow \infty$, and all quantities in Equation (51) apart from $\beta$ become zero.

The second is when the system is initially in equilibrium with $\beta(t=0)=\beta(t \rightarrow \infty)=$ $\frac{\gamma}{2 D}$ and $\left\langle x_{0}\right\rangle=0$ and evolve in time as it is driven out of equilibrium by $u \neq 0$. As $u$ does not affect variance, $\beta(t)=\beta_{0}=\frac{\gamma}{2 D}\left(\partial_{t} \sigma=0\right)$ and $\dot{S}=0$ for all time. In this case, we find

$$
\begin{aligned}
& D \dot{S}_{T}=\dot{Q}=u^{2}\left(1-e^{-\gamma t}\right)^{2}=(\sigma \Gamma)^{2}, \dot{W}=u^{2}\left(1-e^{-\gamma t}\right), \\
& D \mathcal{K}\left[p(x, t) \mid p_{s}(x, t)\right]=\frac{u^{2}}{2 \gamma}\left(1-e^{-\gamma t}\right)^{2}, \\
& D \frac{d}{d t} \mathcal{K}\left[p(x, t) \mid p_{s}(x, t)\right]=u^{2}\left(1-e^{-\gamma t}\right) e^{-\gamma t} .
\end{aligned}
$$

Equation (52) shows that $\dot{S}_{T}, \dot{Q}, \Gamma^{2}, \dot{W}$, and $\mathcal{K}$ start with zero values at $t=0$ and monotonically increase to their asymptotic values as $t \rightarrow \infty$.

Finally, both cases considered above in Equations (51) and (52) have $\partial_{t} \sigma=0$ and thus recover Equation (30):

$$
\Gamma^{2}=\frac{D \dot{S}_{T}}{\sigma^{2}}=\frac{\dot{Q}}{\sigma^{2}}
$$

\section{Inequalities}

Section 4 utilized the average (first moment) of a variable (e.g., $\langle V\rangle$ ) and the average of its first time derivative $\left(\left\langle\partial_{t} V\right\rangle=\dot{W}\right)$ while the work $W=\int d t \dot{W}$ is defined by the time integral of $\dot{W}=\left\langle\partial_{t} V\right\rangle$ in Equation (33). This section aims to show that the rates at which average quantities vary with time are bounded by fluctuations and $\Gamma$. Since the average and time derivatives do not commute, we pay particular attention to when the average is taken.

To this end, let us first define the microscopic free energy $\mu=V+D \ln p$ (called the chemical potential energy in [113]). In terms of $\mu$, we have $J=-p \partial_{x} \mu$ and $\langle\mu\rangle=$ $U-D S=\mathcal{F}$. On the other hand,

$$
\partial_{t} \mu=\partial_{t} V+D \frac{\dot{p}}{p}, \quad\left\langle\partial_{t} \mu\right\rangle=\left\langle\partial_{t} V\right\rangle=\dot{W} .
$$

$\left\langle\partial_{t} \mu\right\rangle=\dot{W}$ means that the average rate of change in the microscopic free is the power. From Equation (54), it follows

$$
\left\langle\left(\partial_{t} \mu-\partial_{t} V\right)^{2}\right\rangle=D^{2} \int d x \frac{\dot{p}^{2}}{p}=D^{2} \Gamma^{2} .
$$

Equation (55) establishes the relation between the microscopic free energy and $\Gamma$. Next, we calculate the time-derivative of $\mathcal{F}$

$$
\frac{d}{d t} \mathcal{F}=\frac{d}{d t}\langle\mu\rangle=\langle\dot{\mu}\rangle+\int d x \mu \dot{p}=\dot{W}+\int d x \mu \dot{p} .
$$

Using $\frac{d}{d t} \mathcal{F}=\dot{W}-D \dot{S}_{T}$ in Equation (56) gives $\dot{S}_{T}$ in terms of $\mu$ as

$$
\dot{W}-\frac{d}{d t} \mathcal{F}=D \dot{S_{T}}=-\int d x \mu \dot{p} .
$$

Equation (57) is to be used in Section 5.1 for linking $\dot{S}_{T}$ to $\Gamma$ through an inequality. 


\subsection{General Inequality Relations}

We now use $\int d x \dot{p}=0, \int d x \dot{p}\langle A\rangle=\langle A\rangle \int d x \dot{p}=0$ for any $A=A(x, t)$ and apply the Schwartz inequality $\left|\int^{t} d t_{1} A\left(t_{1}\right) B\left(t_{1}\right)\right| \leq\left[\int^{t} d t_{1} A^{2}\left(t_{1}\right)\right]^{\frac{1}{2}}\left[\int^{t} d t_{1} B^{2}\left(t_{1}\right)\right]^{\frac{1}{2}}$ to Equations (21), (34) and (57) to obtain

$$
\begin{aligned}
|\dot{S}| & =\left|\int d x \dot{p} \ln p\right| \leq \Gamma\left[\int d x p(\delta \ln p)^{2}\right]^{1 / 2}, \\
|\dot{Q}| & =\left|\int d x V \dot{p}\right| \leq \Gamma\left[\int d x p(\delta V)^{2}\right]^{1 / 2}, \\
D \dot{S}_{T} & =\left|\int d x \mu \dot{p}\right| \leq \Gamma\left[\int d x p(\delta \mu)^{2}\right]^{1 / 2} .
\end{aligned}
$$

Equation (60) (Equation (59)) establishes the inequality between entropy production rate (heat flux) and the product of the RMS fluctuations of the microscopic free energy (potential energy) and $\Gamma$. Since $\delta \mu=\delta V+D(\delta \ln p$ ), we have

$$
\left\langle(\delta \mu)^{2}\right\rangle=\left\langle(\delta V)^{2}\right\rangle+D^{2}\left\langle(\delta \ln p)^{2}\right\rangle+2 D\langle\delta V \delta \ln p\rangle .
$$

These relations are to be used in Section 5.2 below.

\subsection{Applications to the Non-Autonomous O-U Process}

For a linear O-U process with $V=\frac{\gamma}{2}(x-v(t))^{2}$ and $f=-\gamma(x-v(t))$ in Equation (14), we use $\left\langle(\delta x)^{2}\right\rangle=\frac{1}{2 \beta},\left\langle(\delta x)^{4}\right\rangle=3\left\langle(\delta x)^{2}\right\rangle=\frac{3}{4 \beta^{2}}$ and $\partial_{t}\langle x\rangle=-\gamma\langle x-v(t)\rangle$ to show

$$
\begin{aligned}
\delta V & =-\partial_{t}\langle x\rangle \delta x+\frac{\gamma}{2}\left[(\delta x)^{2}-\frac{1}{2 \beta}\right] \\
\left\langle(\delta V)^{2}\right\rangle & =\left(\partial_{t}\langle x\rangle\right)^{2} \sigma^{2}+\gamma^{2} \frac{\sigma^{4}}{2}, \\
\delta \ln p & =\frac{1}{2}-\beta(\delta x)^{2}, \\
\left\langle(\delta \ln p)^{2}\right\rangle & =\frac{1}{2} \\
\langle(\delta \ln p)(\delta V)\rangle & =-\frac{\gamma}{4 \beta} .
\end{aligned}
$$

Using Equations (61)-(62) in Equations (58)-(60) together with $2 D \beta-\gamma=-\frac{\partial_{t} \beta}{2 \beta}$ and $\frac{\partial_{t} \beta}{\beta}=-2 \frac{\partial_{t} \sigma}{\sigma}$ leads to

$$
\begin{aligned}
|\dot{S}| & \leq \frac{1}{\sqrt{2}} \Gamma \\
|\dot{Q}| & \leq \Gamma \sigma\left[\left(\partial_{t}\langle x\rangle\right)^{2}+\frac{\gamma^{2} \sigma^{2}}{2}\right]^{\frac{1}{2}}, \\
D \dot{S}_{T} & \leq \Gamma \sigma\left[\left(\partial_{t}\langle x\rangle\right)^{2}+\frac{1}{2}\left(\partial_{t} \sigma\right)^{2}\right]^{\frac{1}{2}} .
\end{aligned}
$$

Finally, it is useful to examine the extreme cases of Equation (63). First, when $\partial_{t} \sigma=0$, Equation (63) holds as an equality as $D \dot{S}_{T}=\left(\partial_{t}\langle x\rangle\right)^{2}=\sigma^{2} \Gamma^{2}$ (see Equation (27)), recovering Equation (28) with $\partial_{t} \sigma=0$. Second, when $\partial_{t}\langle x\rangle=0$, Equation (63) again holds as an equality since $D \dot{S}_{T}=\left(\partial_{t} \sigma\right)^{2}$ and $\Gamma \sqrt{\frac{1}{2} \sigma^{2}\left(\partial_{t} \sigma\right)^{2}}=\left(\partial_{t} \sigma\right)^{2}$. 


\section{Geodesics, Control and Hyperbolic Geometry}

The section aims to discuss geodesics in information geometry and its implications for self-organization and control. To illustrate the key concepts, we utilize an analytically solvable, generalized $\mathrm{O}-\mathrm{U}$ process given by

$$
\frac{d x}{d t}=-\gamma(t)[x-v(t)]+\xi,
$$

where $\gamma(t)>0$ is a damping constant; $v(t)$ is a deterministic force which determines the time evolution of the mean value of $x$; $\xi$ is a short (delta)-correlated noise with the time-dependent amplitude $D(t)$ in general, satisfying Equation (15).

For the initial condition in Equation (16), the mean value $\langle x\rangle \equiv y(t)$ and $\beta(t)$ are given by

$$
\begin{aligned}
y(t) & =\langle x\rangle=x_{0} e^{-\int_{0}^{t} d t_{1} \gamma\left(t_{1}\right) d t_{1}}+\int_{0}^{t} d t_{1} e^{-\int_{0}^{t} d t_{1}\left[\gamma\left(t_{1}\right)-\gamma(t)\right] d t_{1}} \gamma\left(t_{1}\right) f\left(t_{1}\right), \\
\frac{1}{2 \beta(t)} & =\left\langle(x-\langle x\rangle)^{2}\right\rangle=\frac{e^{-2 \int_{0}^{t} d t_{1} \gamma\left(t_{1}\right) d t_{1}}}{2 \beta_{0}}+\int_{0}^{t} d t_{1} e^{-2 \int_{0}^{t} d t_{1}\left[\gamma\left(t_{1}\right)-\gamma(t)\right] d t_{1}} 2 D\left(t_{1}\right),
\end{aligned}
$$

where $x_{0}=\langle x(t=0)\rangle$.

\subsection{Geodesics-Shortest-Distance Path}

A geodesics between the two spatial locations is a unique path with the shortest distance. A similar concept can be applied to information geometry to define a unique evolution path between the two given PDFs, say, $p\left(x, t_{1}\right)$ and $p\left(x, t_{2}\right)$ in the statistical space. The Wootters' distance in quantum mechanics in Equation (4) is such an example. For time-varying stochastic processes, there is an infinite number of different trajectories between the two PDFs at different times. The key question that we address in this section is how to find an exact time evolution of $p(x, t)$ when initial and final PDFs [15] are given. This is a much more difficult problem than finding a minimum distance between two PDFs (like the Wootter's distance). In the following, we sketch some main steps needed for finding such a unique evolution path (the so-called geodesics) between given initial and final PDFs by minimizing $\mathcal{L}$ (see [15] for detailed steps).

For the O-U process in Equation (64), a geodesic solution does not exist for constant $\gamma$, $v(t)$ and $D$. Thus, finding a geodesic solution boils down to determing suitable functions of $\gamma(t), v(t)$ or $D(t)$ [15]. To be specific, let $p\left(x, t_{0}\right)$ and $p\left(x, t_{F}\right)$, respectively, be the PDFs at the time $t=t_{0}$ and $t_{F}\left(>t_{0}\right)$ and find a geodesic solution by minimizing $\mathcal{L}(t)=$ $\int_{t_{0}}^{t_{F}} d t^{\prime} \Gamma\left(t^{\prime}\right)$. The latter is equivalent to minimizing $\int_{t_{0}}^{t_{F}} d t^{\prime} \mathcal{E}\left(t^{\prime}\right)$ and to keeping $\Gamma$ constant. (This geodesics is also called an optimal path (e.g., see [107]).) We rewrite $\mathcal{E}$ in Equation (27) for the O-U process in terms of $y=\langle x\rangle$

$$
\mathcal{E}=\frac{1}{2 \beta^{2}}\left(\frac{d \beta}{d t}\right)^{2}+2 \beta\left(\frac{d y}{d t}\right)^{2} .
$$

The Euler-Lagrange equation

$$
0=\frac{d \mathcal{E}}{d \beta}-\frac{d}{d t} \frac{d \mathcal{E}}{d \dot{\beta}}, 0=\frac{d \mathcal{E}}{d y}-\frac{d}{d t} \frac{d \mathcal{E}}{d \dot{y}}
$$

$\left(\dot{\beta}=\frac{d \beta}{d t}\right.$ and $\left.\dot{y}=\frac{d y}{d t}\right)$ then gives us

$$
\begin{aligned}
& \frac{d^{2} \beta}{d t^{2}}-\frac{1}{\beta}\left(\frac{d \beta}{d t}\right)^{2}-2 \beta^{2}\left(\frac{d y}{d t}\right)^{2}=0, \\
& \frac{d}{d t}\left[\beta \frac{d y}{d t}\right]=0 \rightarrow \beta \frac{d y}{d t}=c,
\end{aligned}
$$


where $c$ is constant. An alternative method of obtaining Equations (69) and (70) is provided in Appendix C. The following equations are obtained from Equations (69) and (70) [15]

$$
\begin{aligned}
\left(\frac{d \beta}{d t}\right)^{2} & =-4 c^{2} \beta+\alpha \beta^{2}, \\
\Gamma^{2} & =\frac{1}{2 \beta^{2}} \dot{\beta}^{2}+2 c^{2} \beta=\frac{\alpha}{2},
\end{aligned}
$$

where $\alpha$ is another (integration) constant. General solutions to Equations (70) and (71) for $c \neq 0$ were found in terms of hyperbolic functions as [15]

$$
\beta(t)=\frac{4 c^{2}}{\alpha} \cosh ^{2}\left[\frac{1}{2} \sqrt{\alpha}(t-A)\right], \quad y(t)=\frac{\sqrt{\alpha}}{2 c} \tanh \left[\frac{1}{2} \sqrt{\alpha}(t-A)\right]-\frac{\sqrt{\alpha}}{2 c}+B,
$$

where $A$ and $B$ are constant.

Equation (73) can be rewritten using $\sigma=(2 \beta)^{-\frac{1}{2}}$ and $z=\frac{y}{\sqrt{2}}$ as follows

$$
\left(z-z_{c}\right)^{2}+\sigma^{2}=R^{2}, z_{c}=\frac{B}{\sqrt{2}}-s R, R=\frac{\Gamma}{2 c},
$$

where $s$ denotes the sign of $c$ so that $s=1$ when $c>0$ while $s=-1$ when $c<0$. Equation (74) is an equation of a circle for the variables $z$ and $\sigma$ with the radius $R$ and the center $z_{c}$, defined in the upper-half plane where $\sigma \geq 0$. Thus, geodesic motions occur along the portions of a circle as long as $c \neq 0$ (as can be seen in Figure 4). A geodesic moves on a circle with a larger radius for a larger information rate $\Gamma$ and speed and vice versus. This manifests the hyperbolic geometry in the upper half Poincare model $[13,117]$ where the half-plane represents $z$ and $\sigma \neq 0$ (see also Appendix D). The constants $c, \alpha, A$, and $B$ determine the coordinate of the center and the radius of the circle $R$. These constants should be fixed by the fixed conditions at the initial $t=0$ and final time $t_{F}$.

Having found the most general form of the geodesic solution for $y(t)$ and $\beta$, the next steps require finding the values of constant values $c, \alpha, A, B$ to satisfy the boundary conditions at $t=t_{0}$ and $t_{F}$, and then finding appropriate $\gamma(t), D(t)$, and $v(t)$ that ensure the geodesic solutions. This means the O-U process should be controlled by $\gamma(t), D(t)$ and $v(t)$ to ensure a geodesic solution.

(a)

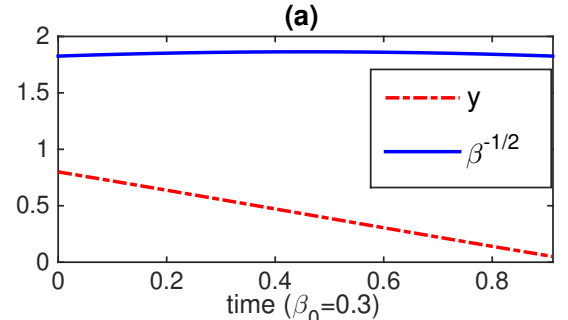

(c)

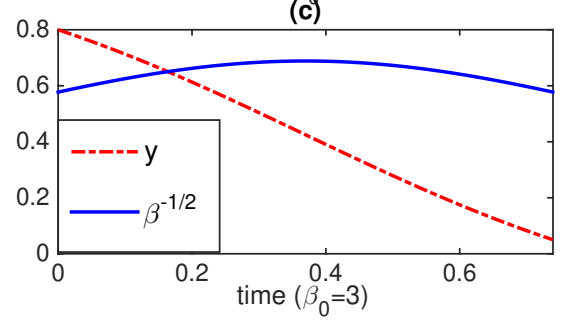

(b)

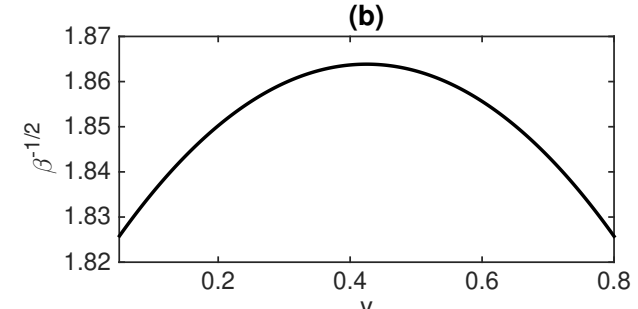

(d)

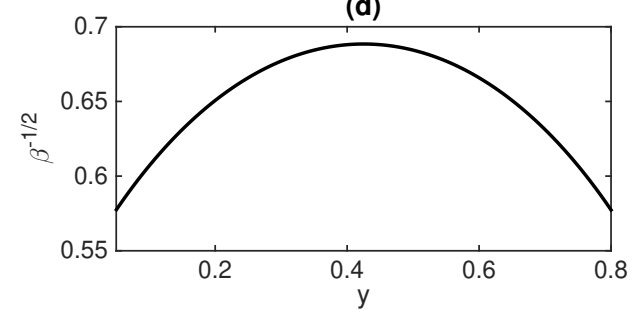

Figure 4. $y$ and $\beta^{-1 / 2}$ against time for $\beta_{0}=0.3$ and 3 in $(\mathbf{a}, \mathbf{c})$, respectively; the corresponding geodesic circular segments in the $\left(y, \beta^{-1 / 2}\right)$ upper half-plane in $(\mathbf{b}, \mathbf{d})$, respectively. In both cases, $y_{0}=\frac{5}{6}$ and $y_{F}=\frac{1}{30}$. (Figure 3 in [15]). 
Figure 4 shows an example of a geodesic solution in the upper half-plane $y$ and $\beta^{-1 / 2}$ when $\gamma(t)=1$ is constant while $D(t)$ and $v(t)$ are time-dependent. The boundary conditions are chosen as $y\left(t_{0}\right)=y_{0}=\frac{5}{6}$ and $y\left(t_{F}\right)=y_{F}=\frac{1}{30}$ in all panels (a) $-(\mathrm{d})$. $\beta\left(t_{0}\right)=\beta_{0}=\beta\left(t_{F}\right)=\beta_{F}=0.3$ in panels (a) and (b) while $\beta_{0}=\beta_{F}=3$ in panels (c) and (d). Interestingly, circular-shape phase-portraits are seen in panels (b) and (d), reflecting hyperbolic geometry noted above (see also Appendix D) $[13,117]$. The speed at which the geodesic motion takes place in the phase portrait is determined by the constant value of $\Gamma=\sqrt{\frac{\alpha}{2}}$ (i.e., the larger $\alpha$, the faster time evolution).

Figure $5 \mathrm{a}, \mathrm{b}$ are the corresponding PDF snapshots at different times (shown in different colors), demonstrating how the PDF evolves from the initial PDF in red to the final PDF in blue. In both cases, it is prominent that the PDF width $\left(\alpha \beta^{-1 / 2}\right)$ initially broadens and then becomes narrower.
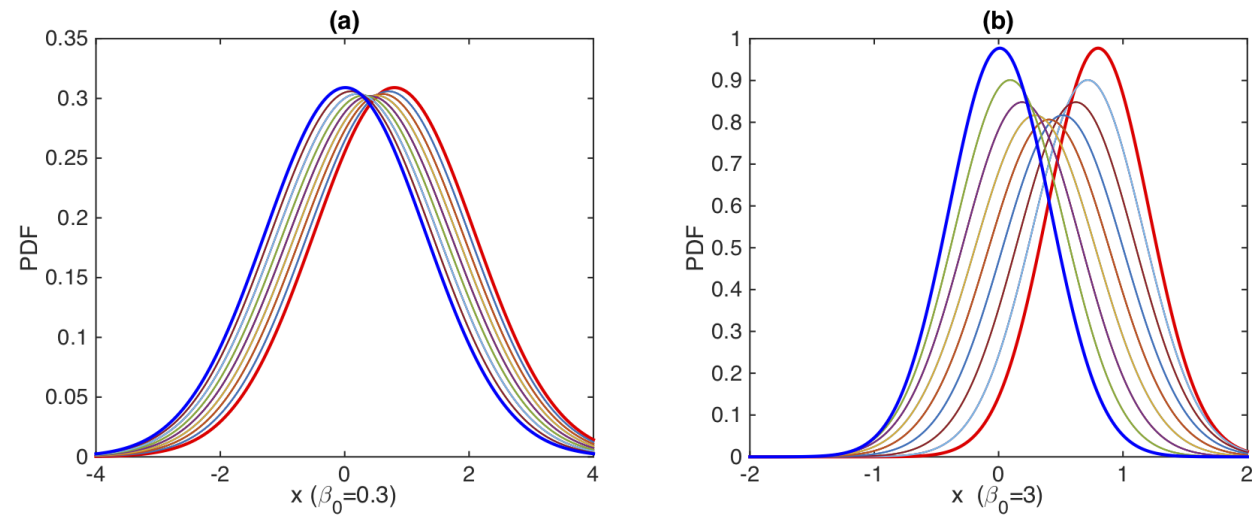

Figure 5. Time evolution of PDFs against $x$ : (a) $\beta_{0}=0.3$ corresponding to Figure $4 \mathrm{a}, \mathrm{b} ;(\mathbf{b}) \beta_{0}=3$ corresponding to Figure $4 \mathrm{c}, \mathrm{d}$. In both cases, $y_{0}=\frac{5}{6}$ and $y_{F}=\frac{1}{30}$. The initial and final PDFs are shown by thick red and blue lines, respectively. (Figure 4a,b in [15]).

\subsection{Comments on Self-Organization and Control}

Self-organization (also called homeostasis) is the novel phenomena where order spontaneously emerges out of disorder and is maintained by different feedbacks in complex systems [45,52,53,118-123]. The extremum principles of thermodynamics such as the minimum entropy production (e.g., $[119,121])$ or maximum entropy entropy production (e.g., [122,123]) have been proposed by considering a steady state or an instant time in different problems.

However, far from equilibrium, self-organization can be a time-varying non-equilibrium process involving perpetual or large fluctuations (e.g., see [52-54]). In this case, the extreme of entropy production should be on accumulative entropy production over time rather than at one instant time nor in a steady state. That is, we should consider the time-integral of the entropy production $\dot{S}_{T}$, or equivalently, the time-integral of $\sqrt{\dot{S}_{T}}$. As seen from Equations (24) and (53), for a linear O-U process with a constant variance, there is an exact proportionality between $\sqrt{\dot{S}_{T}}$ and $\Gamma$. In this case, the extreme of $\mathcal{L}(t)=\int^{t} d t_{1} \Gamma\left(t_{1}\right)$ would be the same as the extreme of $\int^{t} d t_{1} \sqrt{\dot{S}_{T}}$. However, as noted previously, $\Gamma \propto \sqrt{\dot{S}_{T}}$ does not hold in general (e.g., see Equation (28)).

With these comments, we now look at the implications of a geodesic for self-organization, in particular, in biosystems. For the very existence and optimal functions of a living organiss, it is critical to minimize the dispersion of its physical states and to maintain its states within certain bounds upon changing conditions [124]. How fast its state changes in time can be quantified by the surprise rate $\partial_{t}\left[\ln (p(x, t)]\right.$. Since $\int d x p(x, t) \partial_{t} \ln (p(x, t))=0$, we use its RMS value $\sqrt{\left\langle\left(\partial_{t} \ln p\right)^{2}\right\rangle}=\Gamma$ (see Equation (9)) and realize that the total change over a finite time interval $\left[t_{0}, t_{F}\right]$ is nothing more than $\mathcal{L}(t)=\int_{t_{0}}^{t_{F}} d t_{1} \Gamma\left(t_{1}\right)$. Thus, minimizing the accumulative/time-integral of the RMS surprise rate is equivalent to minimizing $\mathcal{L}$. Envisioning surprise rate as biological cost associated with changes (e.g., needed in updating 
the future prediction based on the current state [124,125]), we can then interpret $\mathcal{L}$ as an accumulative biological cost. Thus, geodesic would be an optimal path that minimizes such an accumulative biological cost.

Ref [15] addressed how to utilize this idea to control populations (tumors). Specifically, the results in Section 6.1 were applied to a nonlinear stochastic growth model (obtained by a nonlinear change of variables of the O-U process), and the geodesic solution in Equation (73) was used to find the optimal protocols $v(t)$ and $D(t)$ in reducing a largesize tumor to a smaller one. Here, in this problem, $D(t)$ represents the heterogeneity of tumor cells (e.g., larger $D$ for metastatic tumor) that can be controlled by gene reprogramming while $v(t)$ models the effect of a drug or radiation that reduces the mean tumor population/size.

\section{Discussions and Conclusions}

There has been a growing interest in information geometry from theoretical and practical considerations. This paper discussed some recent developments in information geometric theory, focusing on time-dependent dynamic aspects of non-equilibrium processes (e.g., time-varying mean value, time-varying variance, or temperature) and their thermodynamic and physical/biological implications.

In Sections 2 and 3, by utilizing a Langevin model of an over-damped stochastic process $x(t)$, we highlighted the importance of a path-dependent distance $\mathcal{L}$ in describing time-varying processes. In Sections 4 and 5 , we elucidated the thermodynamic meanings of the relative entropy and the information rate $\Gamma$ by relating them to the entropy production rate $\left(\dot{S}_{T}\right), \dot{S}$, heat flux $\left(Q=D \dot{S}_{m}\right)$, dissipated work $\left(\dot{W}_{D}\right)$, etc., and demonstrated the role of $\Gamma$ in determining bounds (or speed limit) on thermodynamical quantities.

Specifically, in the O-U process, we showed the exact relation $\Gamma=\sqrt{\frac{D}{\sigma^{2}} \dot{S}_{T}+\dot{S}^{2}}$ (Equation (28)), which is simplified as $\sigma \Gamma=\sqrt{D \dot{S}_{T}}$ when $\partial_{t} \sigma=0$ $\left(\sigma=\sqrt{\left\langle(\delta x)^{2}\right\rangle}\right.$ is the standard deviation of $\left.x\right)$. Finally, Section 6 discussed geodesic and its implication for self-organization as well as the underlying hyperbolic geometry. It remains future works to explore the link between $\Gamma$ and the entropy production rate in other (e.g., nonlinear) systems consisting of three or more interacting components or data from self-organizing systems (e.g., normal brain).

Funding: This research received no funding.

Data Availability Statement: Data are available from the author.

Acknowledgments: Eun-jin Kim acknowledges the Leverhulme Trust Research Fellowship (RF2018-142-9) and thanks the collaborators, especially, James Heseltine who contributed to the works in this paper.

Conflicts of Interest: The author declares no conflicts of interest.

\section{Appendix A}

In this Appendix, we show the invariance of Equation (9) when $x$ changes as $y=F(x)$. Using the conservation of the probability, we then have

$$
p(y, t)=p(x, t)\left|\frac{d x}{d y}\right|=p(x, t)\left|\frac{d F(x)}{d x}\right|^{-1},
$$

Since $\frac{d F(x)}{d x}$ is independent of time $t$, it follows that $\partial_{t} p(y, t)=\left[\partial_{t} p(x, t)\right]\left|\frac{d F(x)}{d x}\right|^{-1}$. Using this and $d y=d x\left|\frac{d F(x)}{d x}\right|$, we have

$$
\int d y \frac{1}{p(y, t)}\left[\frac{\partial p(y, t)}{\partial t}\right]^{2}=\int d x \frac{1}{p(x, t)}\left[\frac{\partial p(x, t)}{\partial t}\right]^{2} .
$$


This shows that $p(x, t)$ and $p(y, t)$ give the same $\Gamma^{2}(t)$.

\section{Appendix B. The Coupled O-U Process}

The coupled O-U process for Figure 3 in Section 3.1 is governed by the Fokker-Planck equation [22]

$$
\begin{aligned}
& \frac{\partial P_{1}}{\partial t}=\frac{\partial}{\partial x}\left[\gamma_{1}(x-\mu) P_{1}\right]+D \frac{\partial^{2} P_{1}}{\partial x^{2}}-f_{0} P_{1}+g_{0} P_{2}, \\
& \frac{\partial P_{2}}{\partial t}=\frac{\partial}{\partial x}\left[\gamma_{2}(x-\mu) P_{2}\right]+D \frac{\partial^{2} P_{2}}{\partial x^{2}}+f_{0} P_{1}-g_{0} P_{2} .
\end{aligned}
$$

Here, $D$ is the strength of a short-correlated Gaussian noise given by Equation (15). These equations are the coupled $\mathrm{O}-\mathrm{U}$ processes with the coupling constants $f_{0}$ and $g_{0}$ through the Dichotomous noise $[110,111]$.

For simplicity, we use $\gamma_{1}=\gamma_{2}=\gamma$ and $f_{0}=g_{0}=\epsilon$ and the following initial conditions

$$
\begin{aligned}
& P_{1}(x, 0)=\frac{1}{2} \sqrt{\frac{\beta_{10}}{\pi}} \exp \left[-\beta_{10} x^{2}\right], \\
& P_{2}(x, 0)=\frac{1}{2} \sqrt{\frac{\beta_{20}}{\pi}} \exp \left[-\beta_{20}\left(x-x_{0}\right)^{2}\right] .
\end{aligned}
$$

The solutions are given by

$$
\begin{aligned}
& P_{1}(x, t)=\frac{1}{4}\left[\sqrt{\frac{\beta_{1}}{\pi}}\left(1+e^{-2 \epsilon t}\right) e^{-\beta_{1} x^{2}}+\sqrt{\frac{\beta_{2}}{\pi}}\left(1-e^{-2 \epsilon t}\right) e^{-\beta_{2}\left(x-e^{-\gamma t} x_{0}\right)^{2}}\right], \\
& P_{2}(x, t)=\frac{1}{4}\left[\sqrt{\frac{\beta_{1}}{\pi}}\left(1-e^{-2 \epsilon t}\right) e^{-\beta_{1} x^{2}}+\sqrt{\frac{\beta_{2}}{\pi}}\left(1+e^{-2 \epsilon t}\right) e^{-\beta_{2}\left(x-e^{-\gamma t} x_{0}\right)^{2}}\right],
\end{aligned}
$$

where

$$
\frac{1}{2 \beta_{m}}=\frac{e^{-2 \gamma t}}{2 \beta_{m 0}}+\frac{D}{\gamma}\left(1-e^{-2 \gamma t}\right),
$$

for $m=1,2$. In the limit of $t \rightarrow \infty, P_{1}$ and $P_{2}$ in Equations (A7) and (A8) approach the same equilibrium distribution

$$
P_{m}(x, t)=\frac{1}{2} \sqrt{\frac{\beta_{m}(t \rightarrow \infty)}{\pi}} e^{-\beta_{m}(t \rightarrow \infty) x^{2}},
$$

where $\beta_{m}(t \rightarrow \infty)=\frac{\gamma}{2 D}$. We note that the total PDF $P=P_{1}+P_{2}$ satisfies the single O-U process where the initial PDF is given by the sum of Equations (A5) and (A6).

Figure 3 is shown for the fixed parameter values $\gamma=0.1, D=1$ and $\epsilon=0.5$, $\beta_{20}=\beta_{10}=0.05=\beta(t \rightarrow \infty)=\frac{\gamma}{2 D}=0.05$. Different values of the initial mean position $x_{0}$ of $P_{2}$ are used to examine how metrics depend on $x_{0}$. As noted in Section 2.2, $P_{1}$ at $t=0$ is chosen to be the same as the final equilibrium state which has the zero mean value and inverse temperature $\beta_{10}=0.05$.

\section{Appendix C. Curved Geometry: The Christoffel and Ricci-Curvature Tensors}

A geodesic solution in Section 6.1 can also be found by solving the geodesic equation in general relativity (e.g., [31,107]). To this end, we let the two parameters be $\lambda^{1}=\langle x\rangle=y$ and $\lambda^{2}=\beta$ and express Equation (A11) in terms of the metric tensor $g_{i j}$ as follows (see also Equation (10))

$$
\mathcal{E}=\sum_{i j} \frac{d \lambda^{i}}{d t} g_{i j} \frac{d \lambda^{j}}{d t},
$$


where

$$
g_{i j}=\left(\begin{array}{cc}
\frac{1}{2 \beta^{2}} & 0 \\
0 & 2 \beta
\end{array}\right), \quad \lambda^{i}=\left(\begin{array}{l}
\beta \\
y
\end{array}\right) .
$$

Note that while $g_{i j}$ is diagonal, the 1-st diagonal component depends on $\beta$ (the second parameter). That is, $g_{i i}$ is not independent of $j$-th parameter for $j \neq i$ in general. From Equation (A12), we can find non-zero components of connection tensor $\Gamma_{i j k}=\frac{1}{2}\left[\partial_{i} g_{j k}+\partial_{j} g_{i k}-\partial_{k} g_{i j}\right]\left(\Gamma_{j k}^{i}=g_{i m} \Gamma_{j k m}\right)$

$$
\Gamma_{11}^{1}=-\frac{1}{\beta}, \Gamma_{22}^{1}=-2 \beta^{2}, \Gamma_{12}^{2}=\Gamma_{21}^{2}=\frac{1}{2 \beta} .
$$

A geodesic equation $\frac{d^{2} \lambda^{i}}{d t^{2}}+\Gamma_{m k}^{i} \frac{d \lambda^{m}}{d t} \frac{d \lambda^{k}}{d t}=0$ in terms of the Christoffel tensors becomes

$$
\begin{aligned}
& \ddot{\beta}+\Gamma_{11}^{1} \dot{\beta}^{2}+\Gamma_{22}^{1} \dot{y}^{2}=0, \\
& \ddot{y}+\Gamma_{12}^{2} \dot{\beta} \dot{y}+\Gamma_{21}^{2} \dot{\beta} \dot{y}=0 .
\end{aligned}
$$

Equations (A13)-(A15) give Equation (70). Note that if $g_{i i}$ is independent of the $\lambda^{j}(j \neq i)$ for all $i$ and $j$, the Christoffel tensors have non-zero values only for $\Gamma_{i i}^{i}$, leading to a much simpler geodesic solution (e.g., see [31]).

Finally, to appreciate the curved geometry associated with this geodesic solution, we proceed to calculate the Riemann curvature tensor $R_{k m n}^{i}=\partial_{m} \Gamma_{n k}^{i}+\Gamma_{m p}^{i} \Gamma_{n k}^{p}-\partial_{n} \Gamma_{m k}^{i}-$ $\Gamma_{n p}^{i} \Gamma_{m k}^{p}$ and the Ricci tensor $R_{i j}=R_{i k j}^{k}$ from Equation (A13) and find the following non-zero components [15]

$$
R_{212}^{1}=-R_{221}^{1}=-\beta, R_{112}^{2}=-R_{121}^{2}=\frac{1}{4 \beta^{2}} .
$$

Non-zero curvature tensors represent that the metric space is curved with a finite curvature. Specifically, we find the Ricci tensor $R_{i j}=R_{i k j}^{k}$ and curvature $R$ :

$$
\begin{array}{r}
R_{11}=-\frac{1}{4 \beta^{2}}, R_{22}=-\beta, \\
R=R_{12}=R_{21}=0, \\
R=g_{i j} R_{i j}=-1
\end{array}
$$

The negative curvature is typical of hyperbolic geometry. Finally, using $R=-1$, we calculate the Einstein field equation

$$
G_{i j}=R_{i j}-\frac{1}{2} R g_{i j}=\left(\begin{array}{cc}
-\frac{1}{4 \beta^{2}} & 0 \\
0 & -\beta
\end{array}\right)+\frac{1}{2}\left(\begin{array}{cc}
\frac{1}{2 \beta^{2}} & 0 \\
0 & 2 \beta
\end{array}\right)=0 .
$$

Since $G_{i j}=8 \pi T_{i j}$ where $T_{i j}$ is the stress-energy tensor, we see that $T_{i j}=0$ for this problem.

\section{Appendix D. Hyperbolic Geometry}

The Hyperbolic geometry in the upper-half plane $[13,117]$ becomes more obvious when Equation (A12) is expressed in terms of the two parameters $\langle x(t)\rangle$ and $\sigma(t)$ where $x$ and $y$ axes represent $\langle x(t)\rangle$ and $\sigma(t)$ with the metric tensor

$$
g_{i j}^{H}=\left(\begin{array}{cc}
\frac{2}{\sigma^{2}} & 0 \\
0 & \frac{1}{\sigma^{2}}
\end{array}\right) .
$$




\section{References}

1. Cover, T.M.; Thomas, J.A. Elements of Information Theory; Wiley: New York, NY, USA, 1991.

2. Parr, T.; Da Costa, L.; Friston, K.J. Markov blankets, information geometry and stochastic thermodynamics. Philos. Trans. R. Soc. A 2019, 378, 20190159. [CrossRef] [PubMed]

3. Oizumi, M.; Tsuchiya, N.; Amari, S. Unified framework for information integration based on information geometry. Proc. Nat. Am. Soc. 2016, 113, 14817. [CrossRef] [PubMed]

4. Kowalski, A.M.; Martin, M.T.; Plastino, A.; Rosso, O.A.; Casas, M. Distances in Probability Space and the Statistical Complexity Setup. Entropy 2011, 13, 1055-1075. [CrossRef]

5. Martin, M.T.; Plastino, A.; Rosso, O.A. Statistical complexity and disequilibrium. Phys. Lett. A 2003, 311, 126-132. [CrossRef]

6. Gibbs, A.L.; Su, F.E. On choosing and bounding probability metrics. Int. Stat. Rev. 2002, 70, 419-435. [CrossRef]

7. Jordan, R.; Kinderlehrer, D.; Otto, F. The variational formulation of the Fokker-Planck equation. SIAM J. Math. Anal. 1998, 29, 1-17. [CrossRef]

8. Takatsu, A. Wasserstein geometry of Gaussian measures Osaka J. Math. 2011, 48 1005-1026.

9. Lott, J. Some geometric calculations on Wasserstein space. Commun. Math. Phys. 2008, 277, 423-437. [CrossRef]

10. Gangbo, W.; McCann, R.J. The geometry of optimal transportation. Acta Math. 1996, 177, 113-161. [CrossRef]

11. Zamir, R. A proof of the Fisher information inequality via a data processing argument. IEEE Trans. Inf. Theory 1998, 44, 1246-1250. [CrossRef]

12. Otto, F.; Villani, C. Generalization of an Inequality by Talagrand and Links with the Logarithmic Sobolev Inequality. J. Funct. Anal. 2000, 173, 361-400. [CrossRef]

13. Costa, S.; Santos, S.; Strapasson, J. Fisher information distance. Discrete Appl. Math. 2015, 197, 59-69. [CrossRef]

14. Ferradans, S.; Xia, G.-S.; Peyré, G.; Aujol, J.-F. Static and dynamic texture mixing using optimal transport. Lecture Notes Comp. Sci. 2013, 7893, 137-148.

15. Kim, E.; Lee, U.; Heseltine, J.; Hollerbach, R. Geometric structure and geodesic in a solvable model of nonequilibrium process Phys. Rev. E 2016, 93, 062127. [CrossRef] [PubMed]

16. Nicholson, S.B.; Kim, E. Investigation of the statistical distance to reach stationary distributions. Phys. Lett. A 2015, 379, 83-88. [CrossRef]

17. Heseltine, J.; Kim, E. Novel mapping in non-equilibrium stochastic processes. J. Phys. A 2016, 49, 175002. [CrossRef]

18. Kim, E.; Hollerbach, R. Signature of nonlinear damping in geometric structure of a nonequilibrium process. Phys. Rev. E 2017, 95, 022137. [CrossRef] [PubMed]

19. Kim, E.; Hollerbach, R. Geometric structure and information change in phase transitions. Phys. Rev. E 2017, 95, 062107. [CrossRef]

20. Kim, E.; Jacquet, Q.; Hollerbach, R. Information geometry in a reduced model of self-organised shear flows without the uniform coloured noise approximation. J. Stat. Mech. 2019, 2019, 023204. [CrossRef]

21. Anderson, J.; Kim, E.; Hnat, B.; Rafiq, T. Elucidating plasma dynamics in Hasegawa-Wakatani turbulence by information geometry. Phys. Plasmas 2020, 27, 022307. [CrossRef]

22. Heseltine, J.; Kim, E. Comparing information metrics for a coupled Ornstein-Uhlenbeck process. Entropy 2019, 21, 775. [CrossRef] [PubMed]

23. Kim, E.; Heseltine, J.; Liu, H. Information length as a useful index to understand variability in the global circulation. Mathematics 2020, 8, 299. [CrossRef]

24. Kim, E.; Hollerbach, R. Time-dependent probability density functions and information geometry of the low-to-high confinement transition in fusion plasma. Phys. Rev. Res. 2020, 2, 023077. [CrossRef]

25. Hollerbach, R; Kim, E.; Schmitz, L. Time-dependent probability density functions and information diagnostics in forward and backward processes in a stochastic prey-predator model of fusion plasmas. Phys. Plasmas 2020, 27, 102301. [CrossRef]

26. Guel-Cortez, A.J.; Kim, E. Information Length Analysis of Linear Autonomous Stochastic Processes. Entropy 2020, $22,1265$. [CrossRef] [PubMed]

27. Guel-Cortez, A.J.; Kim, E. Information geometric theory in the prediction of abrupt changes in system dynamics. Entropy 2021, 23, 694. [CrossRef]

28. Kim, E. Investigating Information Geometry in Classical and Quantum Systems through Information Length. Entropy 2018, 20, 574. [CrossRef]

29. Kim, E. Information geometry and non-equilibrium thermodynamic relations in the over-damped stochastic processes. J. Stat. Mech. Theory Exp. 2021, 2021, 093406. [CrossRef]

30. Parr, T.; Da Costa, L.; Heins, C.; Ramstead, M.J.D.; Friston, K.J. Memory and Markov Blankets. Entropy 2021, 23, 1105. [CrossRef]

31. Da Costa, L. ; Thomas, P.; Biswa, S.; Karl, F.J. Neural Dynamics under Active Inference: Plausibility and Efficiency of Information Processing. Entropy 2021, 23, 454. [CrossRef]

32. Frieden, B.R. Science from Fisher Information; Cambridge University Press: Cambridge, UK, 2004.

33. Wootters, W. Statistical distance and Hilbert-space. Phys. Rev. D 1981, 23, 357-362. [CrossRef]

34. Ruppeiner, G. Thermodynamics: A Riemannian geometric model. Phys. Rev. A. 1079, 20, 1608. [CrossRef]

35. Salamon, P.; Nulton, J.D.; Berry, R.S. Length in statistical thermodynamics. J. Chem. Phys. 1985, 82, 2433-2436. [CrossRef]

36. Nulton, J.; Salamon, P.; Andresen, B.; Anmin, Q. Quasistatic processes as step equilibrations. J Chem. Phys. 1985, 83, 334. [CrossRef] 
37. Braunstein, S.L.; Caves, C.M. Statistical distance and the geometry of quantum states. Phys. Rev. Lett. 1994, 72, 3439. [CrossRef]

38. Diósi, L.; Kulacsy, K.; Lukács, B.; Rácz, A. Thermodynamic length, time, speed, and optimum path to minimize entropy production. J. Chem. Phys. 1996, 105, 11220. [CrossRef]

39. Crooks, G E. Measuring thermodynamic length. Phys. Rev. Lett. 2007, 99, 100602. [CrossRef]

40. Salamon, P.; Nulton, J.D.; Siragusa, G.; Limon, A.; Bedeaus, D.; Kjelstrup, D. A Simple Example of Control to Minimize Entropy Production. J. Non-Equilib. Thermodyn. 2002, 27, 45-55. [CrossRef]

41. Feng, E.H.; Crooks, G.E. Far-from-equilibrium measurements of thermodynamic length. Phys. Rev. E. 2009, 79, 012104. [CrossRef]

42. Sivak, D.A.; Crooks, G.E. Thermodynamic Metrics and Optimal Paths. Phys. Rev. Lett. 2012, 8, 190602. [CrossRef] [PubMed]

43. Matey, A.; Lamberti, P.W.; Martin, M.T.; Plastron, A. Wortters' distance resisted: A new distinguishability criterium. Eur. Rhys. J. D 2005, 32, 413-419.

44. d'Onofrio, A. Fractal growth of tumors and other cellular populations: linking the mechanistic to the phenomenological modeling and vice versa. Chaos Solitons Fractals 2009, 41, 875. [CrossRef]

45. Newton, A.P.L.; Kim, E.; Liu, H.-L. On the self-organizing process of large scale shear flows. Phys. Plasmas 2013, $20,092306$. [CrossRef]

46. Kim, E.; Liu, H.-L.; Anderson, J. Probability distribution function for self-organization of shear flows. Phys. Plasmas 2009, 16, 0552304. [CrossRef]

47. Kim, E.; Diamond, P.H. Zonal flows and transient dynamics of the L-H transition. Phys. Rev. Lett. 2003, 90, 185006. [CrossRef] [PubMed]

48. Feinberg, A.P.; Irizarry, R.A. Stochastic epigenetic variation as a driving force of development, evolutionary adaptation, and disease. Proc. Natl. Acad. Sci. USA 2010, 107, 1757. [CrossRef] [PubMed]

49. Wang, N.X.; Zhang, X.M.; Han, X.B. The effects of environmental disturbances on tumor growth. Braz. J. Phys. 2012, $42,253$. [CrossRef]

50. Lee, J.; Farquhar, K.S.; Yun, J.; Frankenberger, C.; Bevilacqua, E.; Yeung, E.; Kim, E.; Balázsi, G.; Rosner, M.R. Network of mutually repressive metastasis regulators can promote cell heterogeneity and metastatic transitions. Proc. Natl. Acad. Sci. USA 2014, 111, E364. [CrossRef] [PubMed]

51. Lee, U.; Skinner, J.J.; Reinitz, J.; Rosner, M.R.; Kim, E. Noise-driven phenotypic heterogeneity with finite correlation time. PLoS ONE 2015, 10, e0132397.

52. Haken, H. Information and Self-Organization: A Macroscopic Approach to Complex Systems, 3rd ed., Springer: Berlin/Heidelberg, Germany, 2006; pp. 63-64.

53. Kim, E. Intermittency and self-organisation in turbulence and statistical mechanics. Entropy 2019, 21, 574. [CrossRef]

54. Aschwanden, M.J.; Crosby, N.B.; Dimitropoulou, M.; Georgoulis, M.K.; Hergarten, S.; McAteer, J.; Milovanov, A.V.; Mineshige, S.; Morales, L.; Nishizuka, N.; et al. 25 Years of Self-Organized Criticality: Solar and Astrophysics. Space Sci. Rev. 2016, 198, 47-166. [CrossRef]

55. Zweben, S.J.; Boedo, J.A.; Grulke, O.; Hidalgo, C.; LaBombard, B.; Maqueda, R.J.; Scarin, P.; Terry, J.L. Edge turbulence measurements in toroidal fusion devices. Plasma Phys. Contr. Fusion 2007, 49, S1-S23. [CrossRef]

56. Politzer, P.A. Observation of avalanche-like phenomena in a magnetically confined plasma. Phys. Rev. Lett. 2000, 84, 1192-1195. [CrossRef] [PubMed]

57. Beyer, P.; Benkadda, S.; Garbet, X.; Diamond, P.H. Nondiffusive transport in tokamaks: Three-dimensional structure of bursts and the role of zonal flows. Phys. Rev. Lett. 2000, 85, 4892-4895. [CrossRef]

58. Drake, J.F.; Guzdar, P.N.; Hassam, A.B. Streamer formation in plasma with a temperature gradient. Phys. Rev. Lett. 1988, 61, 2205-2208. [CrossRef]

59. Antar, G.Y.; Krasheninnikov, S.I.; Devynck, P.; Doerner, R.P.; Hollmann, E.M.; Boedo, J.A.; Luckhardt, S.C.; Conn, R.W. Experimental evidence of intermittent convection in the edge of magnetic confinement devices. Phys. Rev. Lett. 2001, 87, 065001. [CrossRef]

60. Carreras, B.A.; Hidalgo, E.; Sanchez, E.; Pedrosa, M.A.; Balbin, R.; Garcia-Cortes, I. van Milligen, B.; Newman, D.E.; Lynch, V.E. Fluctuation-induced flux at the plasma edge in toroidal devices. Phys. Plasmas 1996, 3, 2664-2672. [CrossRef]

61. De Vries, P.C.; Johnson, M.F.; Alper, B.; Buratti, P.; Hender, T.C.; Koslowski, H.R.; Riccardo, V. JET-EFDA Contributors, Survey of disruption causes at JET. Nucl. Fusion 2011, 51, 053018. [CrossRef]

62. Kates-Harbeck, J.; Svyatkovskiy, A.; Tang, W. Predicting disruptive instabilities in controlled fusion plasmas through deep learning. Nature 2019, 568, 527. [CrossRef]

63. Landau, L.; Lifshitz, E.M. Statistical Physics: Part 1. In Course of Theoretical Physics; Elsevier Ltd.: New York, NY, USA, 1980; Volume 5.

64. Shannon, C.E. A Mathematical Theory of Communication. Bell Syst. Tech. J. 1948, 27, 623. [CrossRef]

65. Jarzynski, C.R. Equalities and Inequalities: Irreversibility and the Second Law of Thermodynamics at the Nanoscale. Annu. Rev. Condens. Matter Phys. 2011, 2, 329-351. [CrossRef]

66. Sekimoto, K. Stochastic Energetic; Lecture Notes in Physics 799; Springer: Heidelberg, Germany, 2010. [CrossRef]

67. Jarzynski, C.R. Comparison of far-from-equilibrium work relations. Physique 2007, 8, 495-506. [CrossRef]

68. Jarzynski, C.R. Nonequilibrium Equality for Free Energy Differences. Phys. Rev. Lett. 1997, 78, 2690-2693. [CrossRef] 
69. Evans, D.J.; Cohen, G.D.; Morriss, G.P. Probability of Second Law Violations in Shearing Steady States. Phys. Rev. Lett. 1993, 71, 2401. [CrossRef]

70. Evans, D.J.; Searles, D.J. The Fluctuation Theorem. Adv. Phys. 2012, 51, 1529-1585. [CrossRef]

71. Gallavotti, G.; Cohen, E.G.D. Dynamical Ensembles in Nonequilibrium Statistical Mechanics. Phys. Rev. Lett. 1995, 74, 2694. [CrossRef]

72. Kurchan, J. Fluctuation theorem for stochastic dynamics. J. Phys. A Math. Gen. 1998, 31, 3719. [CrossRef]

73. Searles, D.J.; Evans, D.J. Ensemble dependence of the transient fluctuation theorem. J. Chem. Phys. 2000, 13, 3503. [CrossRef]

74. Seifert, U. Entropy production along a stochastic trajectory and an integral fluctuation theorem. Phys. Rev. Lett. 2005, 95, 040602. [CrossRef] [PubMed]

75. Abreu, D.; Seifert, U. Extracting work from a single heat bath through feedback. EuroPhys. Lett. 2011, 94 10001. [CrossRef]

76. Seifert, U. Stochastic thermodynamics, fluctuation theorems and molecular machines. Rep. Prog. Phys. 2012, 75, 26001. [CrossRef]

77. Spinney, R.E.; Ford, I.J. Fluctuation relations: A pedagogical overview. arXiv 2012, arXiv:1201.6381S.

78. Haas, K.R.; Yang, H.; Chu, J.-W. Trajectory Entropy of Continuous Stochastic Processes at Equilibrium. J. Phys. Chem. Lett. 2014, 5, 999. [CrossRef]

79. Van den Broeck, C. Stochastic thermodynamics: A brief introduction. Phys. Complex Colloids 2013, 184, $155-193$.

80. Murashita, Y. Absolute Irreversibility in Information Thermodynamics. arXiv 2015, arXiv:1506.04470.

81. Tomé, T. Entropy Production in Nonequilibrium Systems Described by a Fokker-Planck Equation. Braz. J. Phys. 2016, 36, 1285-1289. [CrossRef]

82. Salazar, D.S.P. Work distribution in thermal processes. Phys. Rev. E 2020, 101, 030101. [CrossRef] [PubMed]

83. Kullback, S. Letter to the Editor: The Kullback-Leibler distance. Am. Stat. 1951, 41, 340-341.

84. Sagawa, T. Thermodynamics of Information Processing in Small Systems; Springer: Berlin/Heidelberg, Germany, 2012.

85. Nielsen, M.A.; Chuang, I.L. Quantum Computation and Quantum Information; Cambridge University Press: Cambridge, UK, 2000.

86. Landauer, R. Irreversibility and heat generation in the computing process. IBM J. Res. Dev. 1961, 5, 183-191. [CrossRef]

87. Bérut, A.; Arakelyan, A.; Petrosyan, A.; Ciliberto, S.; Dillenschneider, R.; Lutz, E. Experimental verification of Landauer's principle linking information and thermodynamics. Nature 2012, 483, 187. [CrossRef]

88. Leff, H.S.; Rex, A.F. Maxwell's Demon: Entropy, Information, Computing; Princeton University Press: Princeton, NJ, USA, 1990.

89. Bekenstein, J.D. How does the entropy/information bound work? Found. Phys. 2005, 35, 1805. [CrossRef]

90. Capozziello, S.; Luongo, O. Information entropy and dark energy evolution. Int. J. Mod. Phys. D 2018, 27, 1850029. [CrossRef]

91. Kawai, R.; Parrondo, J.M.R.; Van den Broeck, C. Dissipation: The phase-space perspective. Phys. Rev. Lett. 2007, $98,080602$. [CrossRef] [PubMed]

92. Esposito, M.; Van den Broeck, C. Second law and Landauer principle far from equilibrium. Europhys. Lett. 2011, 95, 40004. [CrossRef]

93. Horowitz, J.; Jarzynski, C.R. An illustrative example of the relationship between dissipation and relative entropy. Phys. Rev. E 2009, 79, 021106. [CrossRef] [PubMed]

94. Parrondo, J.M.R.; van den Broeck, C.; Kawai, R. Entropy production and the arrow of time. New J. Phys. 2009, 11, 073008. [CrossRef]

95. Deffner, S.; Lutz, E. Information free energy for nonequilibrium states. arXiv 2012, arXiv:1201.3888.

96. Horowitz, J.M.; Sandberg, H. Second-law-like inequalities with information and their interpretations. New J. Phys. 2014, 16, 125007. [CrossRef]

97. Nicholson, S.B.; García-Pintos, L.P.; del Campo, A.; Green, J.R. Time-information uncertainty relations in thermodynamics. Nat. Phys. 2020, 16, 1211-1215. [CrossRef]

98. Flego, S.P.; Frieden, B.R.; Plastino, A.; Plastino, A.R.; Soffer, B.H. Nonequilibrium thermodynamics and Fisher information: Sound wave propagation in a dilute gas. Phys. Rev. E 2003, 68, 016105. [CrossRef]

99. Carollo, A.; Spagnolo, B.; Dubkov, A.A.; Valenti, D. On quantumness in multi-parameter quantum estimation. J. Stat. Mech. Theory E 2019, 2019, 094010. [CrossRef]

100. Carollo, A.; Valenti, D.; Spagnolo, B. Geometry of quantum phase transitions. Phys. Rep. 2020, 838, 1-72 . [CrossRef]

101. Davies, P. Does new physics lurk inside living matter?. Phys. Today 2020, 73, 34. [CrossRef]

102. Sjöqvist, E. Geometry along evolution of mixed quantum states. Phys. Rev. Res. 2020, 2, 013344. [CrossRef]

103. Briët, J.; Harremoës, P. Properties of classical and quantum Jensen-Shannon divergence. Phys. Rev. A 2009, 79, 052311. [CrossRef]

104. Casas, M.; Lambertim, P.; Lamberti, P.; Plastino, A.; Plastino, A.R. Jensen-Shannon divergence, Fisher information, and Wootters' hypothesis. arXiv 2004, arXiv:quant-ph/0407147.

105. Sánchez-Moreno, P.; Zarzo, A.; Dehesa, J.S. Jensen divergence based on Fisher's information. J. Phys. A Math. Theor. 2012, 45, 125305. [CrossRef]

106. López-Ruiz, L.; Mancini, H.; Calbet, X. A statistical measure of complexity. Phys. Lett. A 1995, 209, 321-326. [CrossRef]

107. Cafaro, C.; Alsing, P.M. Information geometry aspects of minimum entropy production paths from quantum mechanical evolutions. Phys. Rev. E 2020, 101, 022110. [CrossRef]

108. Ashida, K.; Oka, K. Stochastic thermodynamic limit on E. coli adaptation by information geometric approach. Biochem. Biophys. Res. Commun. 2019, 508, 690-694. [CrossRef]

109. Risken, H. The Fokker-Planck Equation: Methods of Solution and Applications; Springer: Berlin, Germany, 1996. 
110. Van Den Brock, C. On the relation between white shot noise, Gaussian white noise, and the dichotomic Markov process. J. Stat. Phys. 1983, 31, 467-483. [CrossRef]

111. Bena, I. Dichotomous Markov Noise: Exact results for out-of-equilibrium systems (a brief overview). Int. J. Mod. Phys. B 2006, 20, 2825-2888. [CrossRef]

112. Onsager, L.; Machlup, S. Fluctuations and Irreversible Processes. Phys. Rev. 2951, 91, 1505-1512. [CrossRef]

113. Parrondo, J.M.R.; de Cisneros, B.J.; Brito R. Thermodynamics of Isothermal Brownian Motors. In Stochastic Processes in Physics, Chemistry, and Biology; Freund, J.A., Pöschel, T., Eds.; Lecture Notes in Physics; Springer: Berlin/Heidelberg, Germany, 2000; Volume 557. [CrossRef]

114. Gaveau, B.; Granger, L.; Moreau, M.; Schulman, L. Dissipation, interaction, and relative entropy. Phys. Rev. E 2014, 89, 032107. [CrossRef] [PubMed]

115. Ignacio A.; Martínez, G.B.; Jordan M.H.; Juan M.R.P. Inferring broken detailed balance in the absence of observable currents. Nat. Commun. 2019, 10, 3542.

116. Roldán, É.; Barral, J.; Martin, P.; Parrondo, J.M.R.; Jülicher, F. Quantifying entropy production in active fluctuations of the hair-cell bundle from time irreversibility and uncertainty relations. New J. Phys. 2021, 23, 083013. [CrossRef]

117. Chevallier, E.; Kalunga, E.; Angulo, J. Kernel Density Estimation on Spaces of Gaussian Distributions and Symmetric Positive Definite Matrices. 2015. Aavailable online: hal.archives-ouvertes.fr/hal-01245712 (accessed on 29 September 2021).

118. Nicolis, G.; Prigogine, I. Self-Organization in Nonequilibrium Systems: From Dissipative Structures to Order through Fluctuations; John Wiley and Son: New York, NY, USA, 1977.

119. Prigogine, I. Time, structure, and fluctuations. Science 1978, 201, 777-785. [CrossRef]

120. Jaynes, E.T. The Minimum Entropy Production Principle. Ann. Rev. Phys. Chem. 1980, 31, 579-601. [CrossRef]

121. Mehdi, N. On the Evidence of Thermodynamic Self-Organization during Fatigue: A Review. Entropy 2020, $22,372$.

122. Dewar, R.C. Information theoretic explanation of maximum entropy production, the fluctuation theorem and self-organized criticality in non-equilibrium stationary states. J. Phys. A. Math. Gen. 2003, 36, 631-641. [CrossRef]

123. Sekhar, J.A. Self-Organization, Entropy Generation Rate, and Boundary Defects: A Control Volume Approach. Entropy 2021, 23, 1092. [CrossRef] [PubMed]

124. Philipp, S.; Thomas, F.; Ray, D.; Friston, K.J. Exploration, novelty, surprise, and free energy minimization. Front. Psychol. 2013, 4, 1-5.

125. Friston, K.J. The free-energy principle: A unified brain theory? Nat. Rev. Neurosci. 2012, 11, 127-138. [CrossRef] [PubMed] 